\title{
Symmetric and asymmetric solitons in linearly coupled Bose-Einstein condensates trapped in optical lattices
}

\author{
Arthur Gubeskys and Boris A. Malomed \\ Department of Interdisciplinary Studies, \\ School of Electrical Engineering \\ Faculty of Engineering, Tel Aviv University \\ Tel Aviv 69978, Israel
}

\begin{abstract}
We study spontaneous symmetry breaking in a system of two parallel quasi-one-dimensional traps (cores), equipped with optical lattices (OLs) and filled with a Bose-Einstein condensate (BEC). The cores are linearly coupled by tunneling (the model may also be interpreted in terms of spatial solitons in parallel planar optical waveguides with a periodic modulation of the refractive index). Analysis of the corresponding system of linearly coupled Gross-Pitaevskii equations (GPEs) reveals that spectral bandgaps of the single GPE split into subgaps. Symmetry breaking in two-component BEC solitons is studied in cases of the attractive (AA) and repulsive (RR) nonlinearity in both traps; the mixed situation, with repulsion in one trap and attraction in the other (RA), is considered too. In all the cases, stable asymmetric solitons are found, bifurcating from symmetric or antisymmetric ones (and destabilizing them), in the AA and RR systems, respectively. In either case, bi-stability is predicted, with a nonbifurcating stable branch, either antisymmetric or symmetric, coexisting with asymmetric ones. Solitons destabilized by the bifurcation tend to rearrange themselves into their stable asymmetric counterparts. In addition to the fundamental solitons, branches of twisted (odd) solitons in the AA system, and twisted bound states of fundamental solitons in both AA and RR systems, are found too. The impact of a phase mismatch, $\Delta$, between the OLs in the two cores is also studied. It is concluded that $\Delta=\pi / 2$ only mildly deforms the picture, while $\Delta=\pi$ changes it drastically, replacing the symmetry-breaking bifurcations by pseudo-bifurcations, with the branch of asymmetric solutions asymptotically approaching its symmetric or antisymmetric counterpart (in the AA and RR system, respectively), rather than splitting off from it. Also considered is a related model, for a binary BEC in a single-core trap with the OL, assuming that the two species (representing different spin states of the same atom) are coupled by linear interconversion. In that case, the symmetry-breaking bifurcations in the AA and RR models switch their character, if the inter-species nonlinear interaction becomes stronger than the intra-species nonlinearity.
\end{abstract}

PACS numbers: 03.75.Lm, 05.45.Yv, 42.65.Tg, 47.20.Ky

\section{INTRODUCTION AND THE MODEL}

Optical lattices (OLs), i.e., periodic potentials induced by the interference of counterpropagating coherent laser beams, is a powerful tool that allows one to support various dynamical patterns in Bose-Einstein condensates (BECs) [1]. In particular, OLs support gap solitons in BEC with repulsive interaction between atoms (the general topic of BEC solitons was reviewed in Ref. [2]). Gap solitons in BEC were predicted theoretically [3, 4] and then created experimentally in an effectively one-dimensional ("cigar-shaped") trap equipped with the OL in the axial direction. In a self-attractive medium, the periodic OL potential makes it possible to localize a soliton at a prescribed spot, and supports multi-soliton complexes, as was first demonstrated in a model of spatial optical solitons in a planar waveguide with a periodic transverse modulation of the refractive index [6]. Similar theoretical results were then reported for solitons in self-attractive BECs 7]. In particular, in the limit case of a very strong OL, the corresponding Gross-Pitaevskii equation (GPE) 8] reduces to a discrete nonlinear Schrödinger (NLS) equation [9].

Another topic of great interest to BEC 10] and nonlinear optics [13] is spontaneous symmetry breaking of nonlinear fields trapped in dual-core traps (alias double-well potentials). In particular, the spontaneous transition from symmetric solitons in the dual-core trap filled with self-attractive condensate to asymmetric solitons is, in the first approximation, tantamount to the formation of asymmetric solitons in dual-core nonlinear optical fibers [14]. In terms of nonlinear optics, several other dual-core settings have been studied theoretically, including ones with quadratic [11] and cubic-quintic [12] nonlinearities.

Our objective is to explore symmetric, antisymmetric and asymmetric families of BEC solitons in the model of a dual-core trap with cores coupled by linear tunneling and equipped with OLs. We consider symmetric systems with attractive or repulsive nonlinearity in each core (to be referred to as AA and RR ones, i.e., "attractive-attractive" and "repulsive-repulsive"). In the AA system, the solitons originate in the 
semi-infinite bandgap of the OL's linear spectrum, while in the RR system there are gap solitons originating in finite bandgaps (we will consider the first two gaps, demonstrating that the linear coupling between the cores splits them into subgaps). In some cases, soliton families extend into Bloch bands separating the gaps, thus becoming embedded solitons [15].

In optics, spontaneous symmetry breaking in two-component gap solitons was studied in models of dualcore [16] and triple-core [17] fiber Bragg gratings (solitons in triangular and planar triple-core optical waveguides without gratings were considered in Refs. [18]). A mixed RA system, with repulsion in one core and attraction in the other, will be considered too (the sign of the interaction may be selectively reversed by means of the Feshbach resonance [19]).

The basic model that will be dealt with in this work amounts to the following system of linearly coupled normalized GPEs for the mean-field BEC wave functions in the two cores, $\psi(x, t)$ and $\phi(x, t)$ :

$$
\begin{array}{r}
i \psi_{t}+\psi_{x x}+\varepsilon \cos (2 x) \psi+g_{1}|\psi|^{2} \psi+\kappa \phi=0 \\
i \phi_{t}+\phi_{x x}+\varepsilon \cos (2 x+\Delta) \phi+g_{2}|\phi|^{2} \phi+\kappa \psi=0
\end{array}
$$

where $\varepsilon$ is the strength of the OL, $g_{1,2}= \pm 1$ are signs of the nonlinearity $\kappa$ is the linear-coupling coefficient, accounted for by the linear tunneling between the cores, and $\Delta$ takes into regard a possible phase shift (mismatch) between the OLs in the coupled cores. The above-mentioned symmetry breaking takes place in the system with $\Delta=0$; a change of the symmetry-breaking bifurcations under the action of the mismatch will be considered too.

In the limit of a very deep OL $(\varepsilon \rightarrow \infty)$, arguments similar to those applied to the single GPE [9] suggest that the symmetric version of Eqs. (1) reduces to a system of two linearly coupled discrete NLS equations. In fact, a system of that type was introduced in Ref. [20], as a model of a photonic-crystal coupler for optical signals.

The use of Eqs. (1) in the effectively one-dimensional (1D) form implies that both cores are subjected to tight transverse confinement, thus allowing one to reduce the underlying three-dimensional GPE to its counterparts in 1D, as elaborated in several works [21]. Then, the temporal and spatial variables in Eqs. (10) are related to physical time $T$ and axial coordinate $X$ by

$$
t \equiv T\left(\pi^{2} \hbar / m d^{2}\right), x \equiv \sqrt{2} \pi X / d
$$

where $m$ is the atomic mass, and $d$ the OL period in physical units. Further, the scaled 1D wave functions are related to their $3 \mathrm{D}$ counterparts, $\Psi$ and $\Phi$, as follows:

$$
\left\{\begin{array}{l}
\Psi(X, R, T) \\
\Phi(X, R, T)
\end{array}\right\}=e^{-i \omega_{\perp} T} \sqrt{\frac{\pi}{2\left|a_{s}\right| d^{2}}}\left\{\begin{array}{c}
\psi(x, t) \\
\phi(x, t)
\end{array}\right\} \exp \left(-\frac{\omega_{\perp} m}{2 \hbar} R^{2}\right),
$$

where $\omega_{\perp}$ and $R$ are the transverse trapping frequency and radial coordinate (around each core), and $a_{s}$ the $s$-wave scattering length of atomic collisions (it is negative if the interaction is attractive). Due to the normalizations, the lattice strength is represented by $\varepsilon=E_{0} / E_{\mathrm{rec}}$, where $E_{\mathrm{rec}}=(\pi \hbar)^{2} /\left(m d^{2}\right)$ is the lattice recoil energy, and $E_{0}$ is the depth of the periodic potential in physical units.

For the experimental realization of the AA and RR systems, respectively, ${ }^{7} \mathrm{Li}$ [22] and ${ }^{87} \mathrm{Rb}$ [1] condensates are appropriate. Results for solitons presented below are in the ballpark of $\kappa \sim 1$ in Eqs. (11). With regard to Eqs. (2), the corresponding tunnel-coupling time, which is $t_{\text {coupl }}=\pi /(2 \kappa)$ in normalized units, translates, for $d=1 \mu \mathrm{m}$, into physical coupling time $T_{\text {coupl }} \sim 10 \mu \mathrm{s}$ and $100 \mu \mathrm{s}$, for lithium and rubidium, respectively. As for the number of atoms in the solitons, in the AA system is scales, in the normalized units, between $N \simeq 2$ and $N \simeq 15$, see Fig. (3) below. As follows from Eqs. (2) and (3), this corresponds to the actual number of ${ }^{7} \mathrm{Li}$ atoms per soliton between $10^{4}$ and $10^{5}$, if experimentally relevant values are assumed, $\omega_{\perp} \simeq 2 \pi \times 1$ $\mathrm{KHz}$ and $a_{s} \simeq-0.15 \mathrm{~nm}$ (in the experiments without the OL, the number of atoms in the solitons was up to 5,000 [22]). Similarly, in the RR model the number of ${ }^{87} \mathrm{Rb}$ atoms in gap solitons is expected to be $\sim 1000$ (in the first experiments, it was $\simeq 250[1]$ ).

Equations (11) may also be interpreted in terms of nonlinear optics, describing spatial distribution of light in two parallel planar waveguides, with periodic transverse modulation of the refractive index, i.e., as a linear-coupled pair of waveguides considered in Ref. [6]. In that case, $t$ is the propagation distance, $x$ is the transverse coordinate, while $\psi$ and $\phi$ are amplitudes of the electromagnetic fields in the planar cores. 


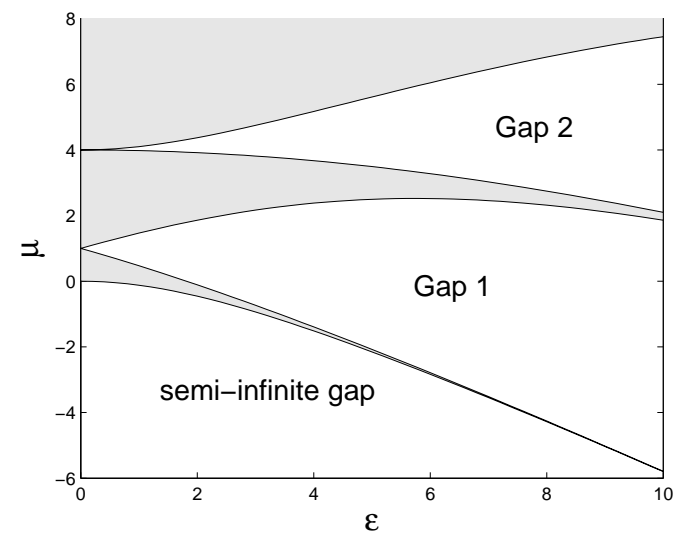

(a)

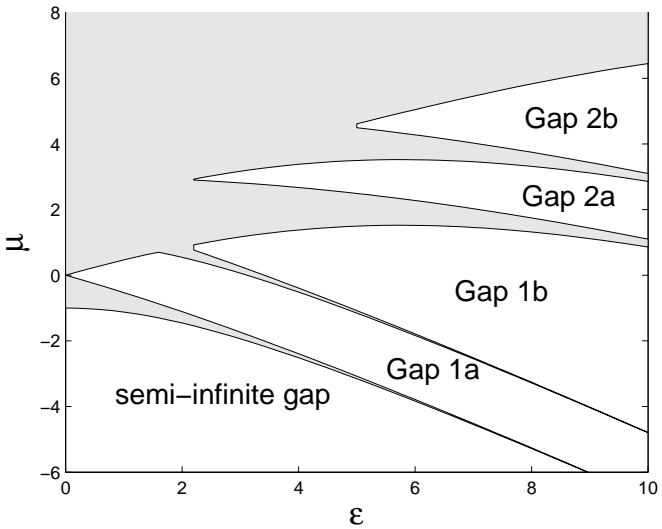

(b)

FIG. 1: Transformation of the linear spectrum with the increase of the lattice strength $\varepsilon$. Here and in other figures including the spectrum, the shaded areas show Bloch bands. (a) The ordinary (decoupled) Mathieu equation, i.e., each equation (4) and (5) with $\kappa=0$; (b) the coupled system, with $\kappa=1$ and $\Delta=0$.

Besides the model based on Eqs. (11), we will also use a modified one, which additionally includes nonlinear coupling between $\psi$ and $\phi$, see Eqs. (17) below. It pertains to a single-core trap equipped with the OL, that contains a BEC mixture of two different spin states of the same atom 23]. The linear-coupling term represents the linear interconversion between the states induced by a spin-flipping electromagnetic wave [24]. While various effects were predicted in the latter setting [25], the spontaneous symmetry breaking in two-component solitons, that we consider in this work, was not studied before.

The paper is organized as follows. In Section II, we analyze the linear spectrum of the coupled system. Methods used for the analysis of soliton solutions are summarized in Section III. Section IV reports results for fundamental (even) and twisted (odd) solitons, as well as bound states of solitons, in symmetric systems, of both the AA and RR types. Results for asymmetric systems (of the RA type, as well as in systems with mismatched OLs) are collected in Section V, including a newly found pseudo-bifurcation, in the system with $\Delta=\pi$. A summary of results obtained in the model which includes nonlinear interaction between the coupled components is given in Section VI, and Section VII concludes the paper.

\section{LINEAR SPECTRUM}

Our first objective is to examine how the linear coupling, accounted for by coefficient $\kappa$ in Eqs. (11), alters the spectrum of the linear system (first, in the model with aligned lattices, $\Delta=0$ ). We look for solutions to the linearized equations as $\{\psi(x, t), \phi(x, t)\}=[\alpha(x) \pm \beta(x)] e^{-i \mu t}$, with chemical potential $\mu$. This leads to decoupled Mathieu equations (ME) with eigenvalues $\mu \pm \kappa$,

$$
\begin{aligned}
\alpha^{\prime \prime}+\varepsilon \cos (2 x) \alpha(x)+(\mu+\kappa) \alpha(x) & =0, \\
\beta^{\prime \prime}+\varepsilon \cos (2 x) \beta(x)+(\mu-\kappa) \beta(x) & =0,
\end{aligned}
$$

where the prime stands for $d / d x$. The ME spectrum gives rise to the well-known bandgap structure. Note that, in the model of two linearly coupled Bragg gratings (i.e., a system of four equations for counterpropagating waves in two cores), the coupling leads to shrinkage or closure of the spectral gap [16]. In the present case, the effect of the coupling is more complex. Given $\mu$ belongs to a bandgap in the present model if $\mu$ falls into one of the gaps in both equations (4) and (5). Further consideration demonstrates that, depending on the values of $\kappa$ and OL strength $\varepsilon$, each gap originating from the ME spectrum either shrinks (or, sometimes, completely closes up), similar to the situation in the above-mentioned model of linearly coupled Bragg gratings, or splits into pairs of subgaps. An example of the splitting of the first two gaps [Gap $\{1,2\}$ $\rightarrow$ Gaps $\{(1 \mathrm{a}, 1 \mathrm{~b}),(2 \mathrm{a}, 2 \mathrm{~b})\}]$ under the action of the linear coupling is displayed in Fig. [

Unlike Eqs. (4) and (5), the linearized equations do not decouple with $\Delta \neq 0$ in Eqs. (1). A typical example of the dependence of the spectrum on $\Delta$ is shown in Fig. 2. With the increase of $\Delta$, the subgaps (that have 


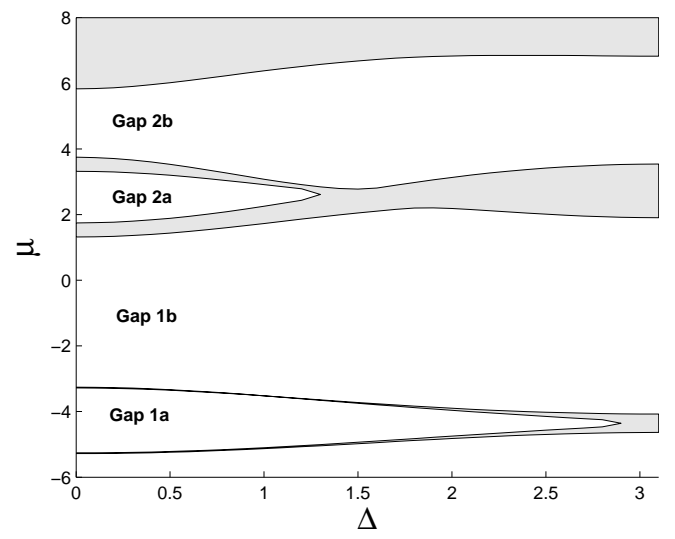

FIG. 2: The transformation of the linear spectrum with the increase of mismatch $\Delta$ for $\varepsilon=8, \kappa=1$.

appeared as a result of the coupling-induced splitting) shrink. At $\Delta$ close to $\pi / 2$, gap 2a disappears, which is followed by the disappearance of gap 1a. At $\Delta=\pi$, the entire spectrum qualitatively resembles that of the ordinary ME.

\section{ANALYSIS OF SOLITON SOLUTIONS}

\section{A. Symmetric, antisymmetric, and asymmetric solitons}

Stationary solutions to Eqs. (11) are looked as $\{\psi, \phi\}=\{u(x), v(x)\} e^{-i \mu t}$, with real functions $u$ and $v$ obeying the equations

$$
\begin{gathered}
\mu u+u^{\prime \prime}+\varepsilon \cos (2 x) u+g_{1} u^{3}+\kappa v=0, \\
\mu v+v^{\prime \prime}+\varepsilon \cos (2 x+\Delta) v+g_{2} v^{3}+\kappa u=0 .
\end{gathered}
$$

First, we explore the symmetries of the system in the AA $\left(g_{1}=g_{2}=+1\right)$ and $\mathrm{RR}\left(g_{1}=g_{2}=-1\right)$ cases with aligned OLs, $\Delta=0$. To this end, we designate $\hat{u}_{0}(x ; \mu)$ the solution of the single-component GPE, i.e. either of two equations (6) with $\kappa=\Delta=0$, corresponding to chemical potential $\mu$. Then, Eqs. (6) give rise to symmetric and antisymmetric solitons,

$$
\begin{gathered}
u(x ; \mu)=v(x ; \mu)=\hat{u}_{0}(x ; \mu+\kappa), \\
u(x ; \mu)=-v(x ; \mu)=\hat{u}_{0}(x ; \mu-\kappa) .
\end{gathered}
$$

If the linear coupling splits the bandgap into two subgaps as outlined above, the shifts of the chemical potential as per Eqs. (7) and (8), $\mu \rightarrow \mu \pm \kappa$, displace the symmetric and antisymmetric soliton to the lower and higher subgap, respectively. In particular, in the coupled AA system, symmetric solitons, which are generated by their counterparts belonging to the semi-infinite gap in the single-component GPE with attraction according to Eq. (77), remain in the semi-infinite gap, while the corresponding anti-symmetric solitons may either stay in the semi-infinite gap, or move to subgap 1a (in the case shown in Fig. 1), or even end up inside the Bloch band separating the semi-infinite gap and subgap 1a. In the latter case, the antisymmetric soliton becomes embedded (into the continuous spectrum), as defined in Refs. [15].

The central issue to be considered below is a possibility of spontaneous symmetry breaking, i.e. finding a bifurcation point beyond which asymmetric solitons emerge. To quantify the symmetry breaking, we define norms in the two cores, and the asymmetry ratio, $\Theta$, as

$$
N_{u, v} \equiv \int_{-\infty}^{+\infty}\left\{u^{2}(x), v^{2}(x)\right\} d x, \Theta \equiv \frac{\left|N_{u}-N_{v}\right|}{N_{u}+N_{v}}
$$

[Eqs. (1) conserve only the total norm, $N \equiv N_{u}+N_{v}$ ]. The bifurcation was found from a numerical solution of Eqs. (6). The results are reported in the next section for the models of the AA, RR, and RA types. 


\section{B. Soliton stability}

To tackle the stability problem, perturbed solutions are taken as

$$
\begin{gathered}
\{\psi(x, t), \phi(x, t)\}=\left\{\left[\hat{u}(x ; \mu)+\xi_{1}(x) e^{i \eta t}\right],\left[\hat{v}(x ; \mu)+\xi_{3}(x) e^{i \eta t}\right]\right\} e^{-i \mu t}, \\
\left\{\psi^{*}(x, t), \phi^{*}(x, t)\right\}=\left\{\left[\hat{u}^{*}(x ; \mu)+\xi_{2}(x) e^{i \eta t}\right],\left[\hat{v}^{*}(x ; \mu)+\xi_{4}(x) e^{i \eta t}\right]\right\} e^{i \mu t},
\end{gathered}
$$

where $\{\hat{u}(x ; \mu), \hat{v}(x ; \mu)\} e^{-i \mu t}$, with real functions $\hat{u}(x ; \mu)$ are $\hat{v}(x ; \mu)$, are stationary solutions of Eqs. (6). Perturbations $\xi_{1,3}$ of fields $\psi, \phi$, and their complex conjugates, $\xi_{2,4}$, are formally treated as independent functions. Substituting expressions (10) in Eqs. (1) and linearizing, we arrive at an eigenvalue problem,

$$
\left[\begin{array}{cc}
\mathcal{L}\left(\hat{u}(x ; \mu), \mu, g_{1}, 0\right) & \kappa \sigma_{3} \\
\kappa \sigma_{3} & \mathcal{L}\left(\hat{v}(x ; \mu), \mu, g_{2}, \Delta\right)
\end{array}\right]\left[\begin{array}{l}
\xi_{12} \\
\xi_{34}
\end{array}\right]=\eta\left[\begin{array}{l}
\xi_{12} \\
\xi_{34}
\end{array}\right],
$$

where $\xi_{12} \equiv\left(\xi_{1}, \xi_{2}\right)^{T}, \xi_{34} \equiv\left(\xi_{3}, \xi_{4}\right)^{T}$, the linear-stability operator for the single-component GPE is

$$
\mathcal{L}(f(x), \mu, g, \Delta)=\left[\frac{d^{2}}{d x^{2}}+\varepsilon \cos (2 x+\Delta)+2 g f^{2}(x)+\mu\right] \sigma_{3}+i g f^{2}(x) \sigma_{2},
$$

and $\sigma_{3}$ and $\sigma_{2}$ are the Pauli matrices. In the single-component GPE, families of fundamental solitons have been shown to be stable [26], i.e., all eigenvalues of the corresponding operator $\mathcal{L}\left(\hat{u}_{0}(x ; \mu), \mu, g, 0\right)$ are real.

Equation (11) can be simplified for symmetric and anti-symmetric solutions. Substituting, respectively, expressions (77) and (8) (with $\Delta=0$ and $g_{1}=g_{2} \equiv g$ ), we arrive at a reduced eigenvalue problem for the symmetric soliton,

$$
\begin{aligned}
& \mathcal{L}\left(\hat{u}_{0}(x ; \mu+\kappa), \mu+\kappa, g, 0\right) \xi_{+}=\eta \xi_{+}, \\
& \mathcal{L}\left(\hat{u}_{0}(x ; \mu+\kappa), \mu-\kappa, g, 0\right) \xi_{-}=\eta \xi_{-}
\end{aligned}
$$

$\left(\xi_{ \pm} \equiv \xi_{12} \pm \xi_{34}\right)$, and its counterpart for the anti-symmetric one,

$$
\begin{aligned}
& \mathcal{L}\left(\hat{u}_{0}(x ; \mu-\kappa), \mu+\kappa, g, 0\right) \xi_{+}=\eta \xi_{+}, \\
& \mathcal{L}\left(\hat{u}_{0}(x ; \mu-\kappa), \mu-\kappa, g, 0\right) \xi_{-}=\eta \xi_{-} .
\end{aligned}
$$

Since Eqs. (13) and (16) are tantamount to the linear-stability problem in the single-component GPE, the stability of the soliton in the latter equation is a necessary condition for the full stability of symmetric and antisymmetric solitons in the coupled system. To identify the full stability conditions for the symmetric and antisymmetric solitons, we solved the additional eigenvalue problems, i.e., respectively, Eqs. (14) and (15). The stability of asymmetric solitons was inferred from a numerical solution of the full eigenvalue problem, based on Eq. (11). The solution is stable if all respective eigenvalues $\eta$ are real.

\section{RESULTS (SYMMETRIC SYSTEMS)}

\section{A. The attraction-attraction model}

Families of numerically found stationary soliton solutions of Eqs. (6) for the AA system $\left(g_{1}=g_{2}=+1\right)$ with zero mismatch $(\Delta=0)$ are displayed in Fig. 3 for $\kappa=1$, in the cases of weak $(\varepsilon=1)$ and strong $(\varepsilon=8)$ OLs. We observe that a branch of asymmetric solitons bifurcates from the symmetric one, while the antisymmetric solutions do not give rise to any bifurcation. We also notice that the antisymmetric branch extends through the Bloch band separating the semi-infinite gap and the first finite bandgap, 1a; as said above, the solitons are embedded ones inside the band. Panels (b) and (d) in the figure clearly show that the symmetry-breaking bifurcation is supercritical (the bifurcating branch immediately goes forward in $-\mu$, without turning backward, the latter being a characteristic feature of the subcritical bifurcation in particular, in the system of linearly coupled NLS equations of the AA type without the lattice potential 14). The present situation is, generally, similar to that in the system of linearly coupled Bragg gratings [16] (where the bifurcation is supercritical too). 


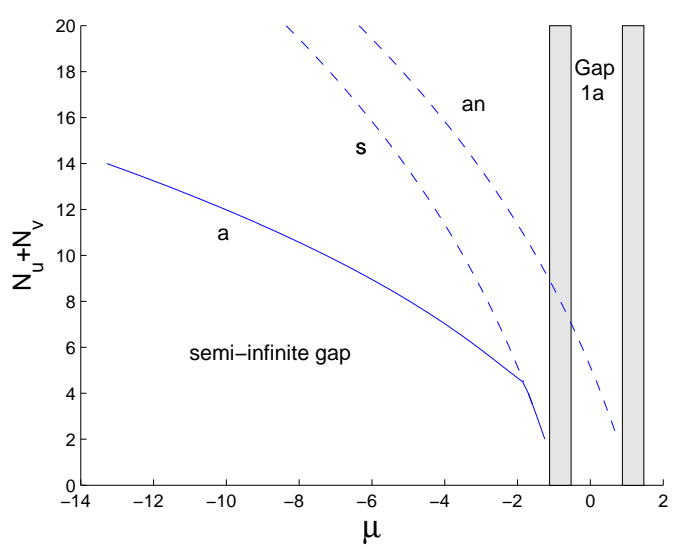

(a)

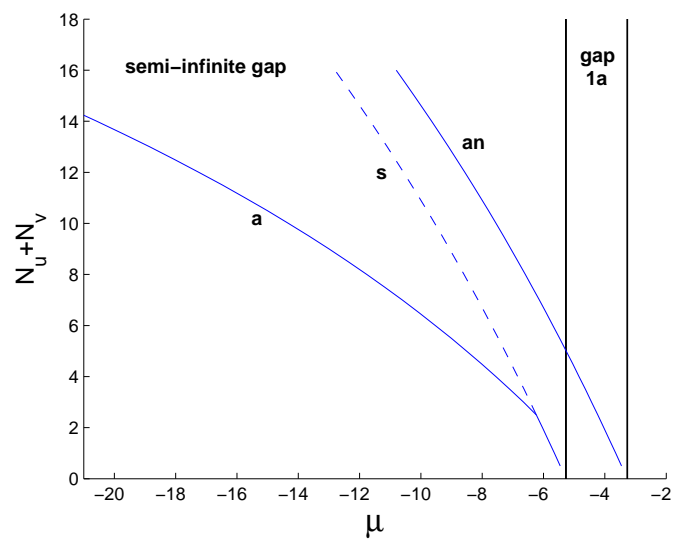

(c)

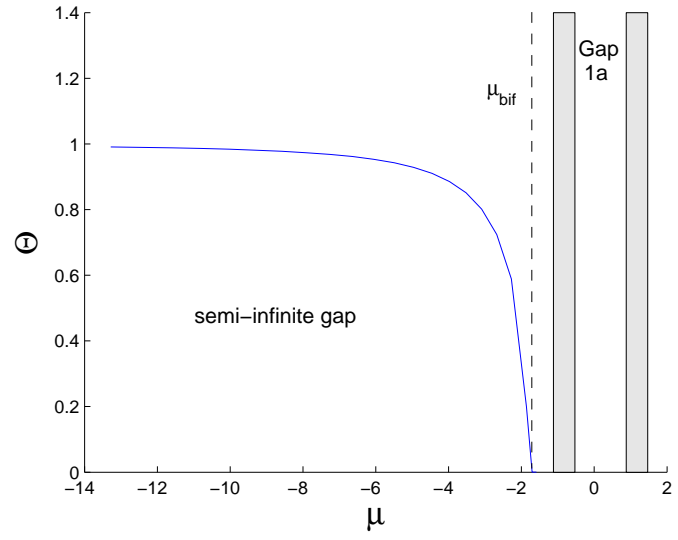

(b)

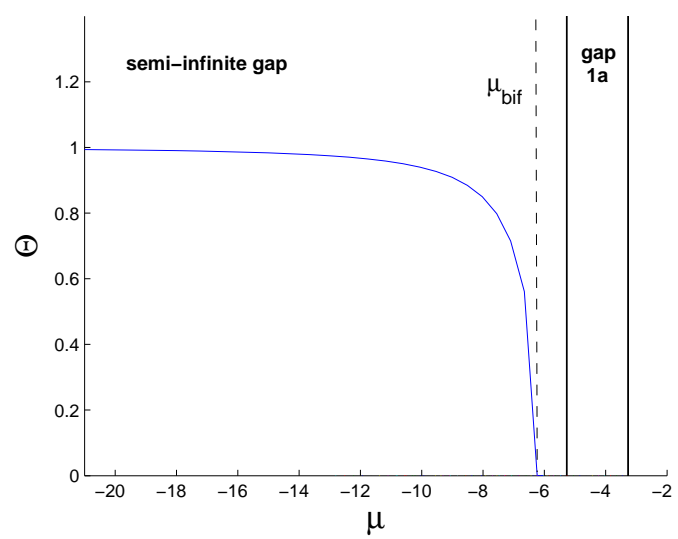

(d)

FIG. 3: (Color online) Soliton families in the attraction-attraction model with $\kappa=1$. In this figure and below, the results are displayed for the dual-core model with aligned lattices $(\Delta=0)$, unless a nonzero value of $\Delta$ is indicated. Presented are cases of relatively weak, $\varepsilon=1$ (a,b), and strong, $\varepsilon=8(\mathrm{c}$, d), lattices. Here and below, stable and unstable branches are shown by solid and dashed lines, with labels "s", "an" and "a" referring to symmetric, antisymmetric, and asymmetric solutions, respectively. (a,c): The total norm of the soliton versus its chemical potential, $\mu$. (b,d): The asymmetry ratio [defined as per Eq. (91)] versus $\mu$, for families of asymmetric solitons; the vertical line labeled $\mu_{\text {bif }}$ marks the bifurcation point. Note that the branch of anti-symmetric solutions is completely unstable in the weak lattice $(\mathrm{a}, \mathrm{b})$, and completely stable in the strong one $(\mathrm{b}, \mathrm{d})$.

The change of the bifurcation with variation of coupling constant $\kappa$ is illustrated by Fig. 4. In particular, the critical value of the coupling constant, $\kappa_{\text {bif }}$, at which the bifurcation occurs is shown in panel (b) as a function of the soliton's total norm [note that in Fig. 3(a), which pertains to $\kappa=1$, the bifurcation in the system with $\varepsilon=1$ occurs at $N \approx 4$, in compliance with Fig. 4(b)]. Naturally, $\kappa_{\text {bif }}$ grows with $N$, as the bifurcation is a result of the competition between the nonlinear and linear properties of the system, accounted for by $N$ and $\kappa$, respectively. On the other hand, the dependence of the bifurcation point on the lattice's depth $\varepsilon$ is quite weak, the stronger lattice making the region of the existence of asymmetric solitons somewhat larger (which can be easily understood too, as the lattice tends to pin and thus stabilize any localized pattern).

The above results bear some implications for stability of the solutions, as, in models of the NLS type with self-focusing, a necessary stability condition is given by the Vakhitov-Kolokolov (VK) criterion [28. In the present notation, it is $d N / d \mu<0$. As seen in Fig. 3. all solution branches satisfy this condition (however, as shown below, not all of them are stable, as the VK criterion is not sufficient for the stability). 


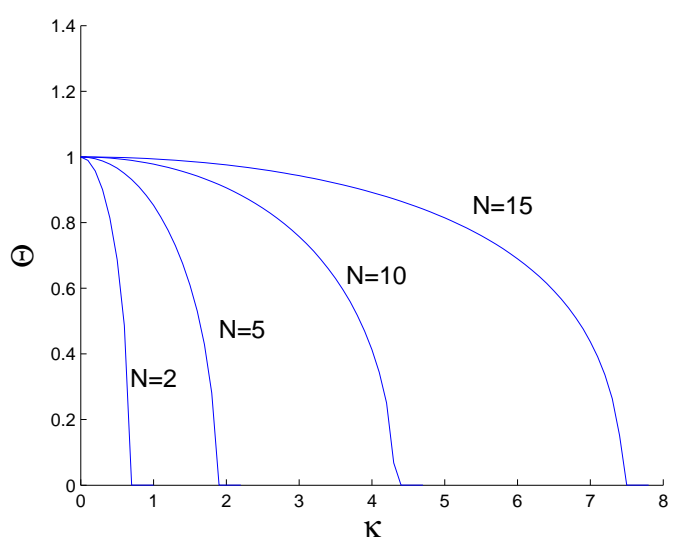

(a)

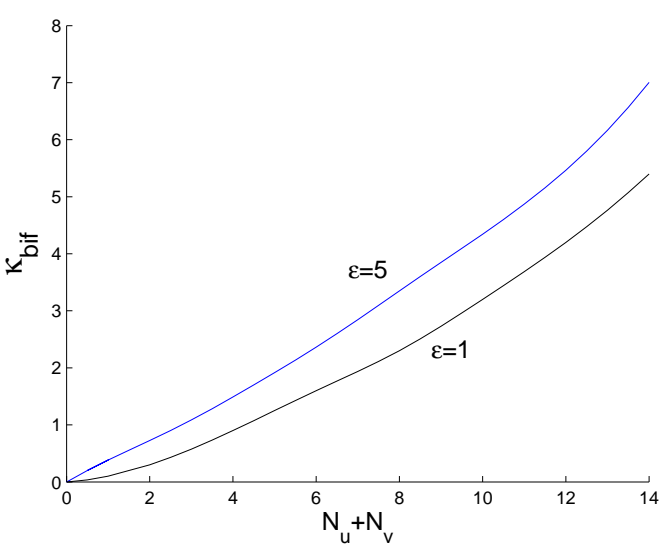

(b)

FIG. 4: (Color online) Properties of asymmetric solitons in the attraction-attraction system. (a) The asymmetry ratio [defined as per Eq. (9)] as a function of coupling coefficient $\kappa$ at $\varepsilon=5$ and different values of the soliton's norm. (b) The value of $\kappa$ at the bifurcation point versus the soliton's norm at different values of the lattice strength, $\varepsilon$.

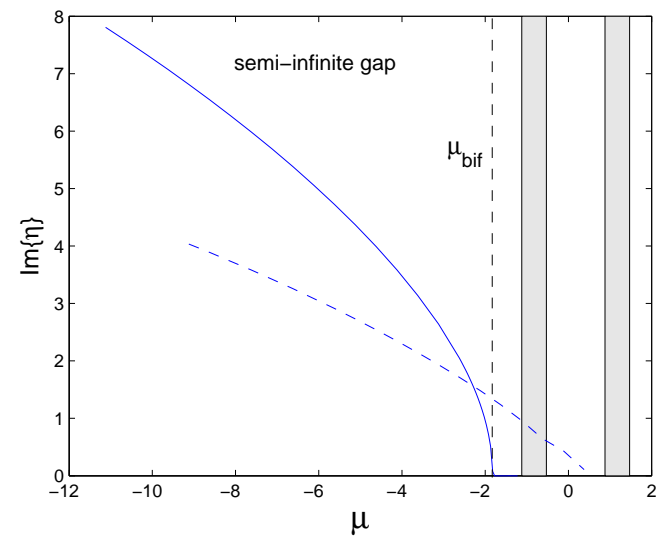

FIG. 5: (Color online) The largest instability growth rates for symmetric and anti-symmetric solitons in the attraction-attraction system, with $\varepsilon=1, \kappa=1$, are shown by the continuous and dashed curves, respectively.

To investigate the stability of the symmetric and antisymmetric soliton families presented in Fig. 3 in an accurate form, we solved the reduced eigenvalue problem, based on Eq. (14) or (15), respectively, to identify the eigenvalue with the largest imaginary part, $\operatorname{Im}\{\eta\}$, i.e., the fastest growing unstable mode. Figure 5 shows the result for a relatively weak OL, with $\varepsilon=1$. We conclude that the symmetric soliton is stable up to the bifurcation point, where the branch of the asymmetric solitons emerges. Beyond this point, the symmetric solitons are unstable. On the other hand, the branch of the anti-symmetric solitons, which undergoes no bifurcation in the AA system, is unstable in weak OLs, see Figs. 3(a) and 5 but becomes stable in a stronger lattice, see Fig. 3(c), In fact, strong OLs give rise to bistability: the antisymmetric soliton coexists, as a stable solution, with either symmetric or asymmetric one. Finally, numerical solution of the full eigenvalue problem, based on Eq. (11), for the asymmetric solitons demonstrates that they are stable whenever they exist.

The predictions of the linear stability analysis have been confirmed by direct numerical simulations of the underlying equations (1). In particular, Fig. [6 shows that unstable symmetric solitons evolve into stable asymmetric ones. It has also been checked that antisymmetric solitons in sufficiently strong OLs are stable 


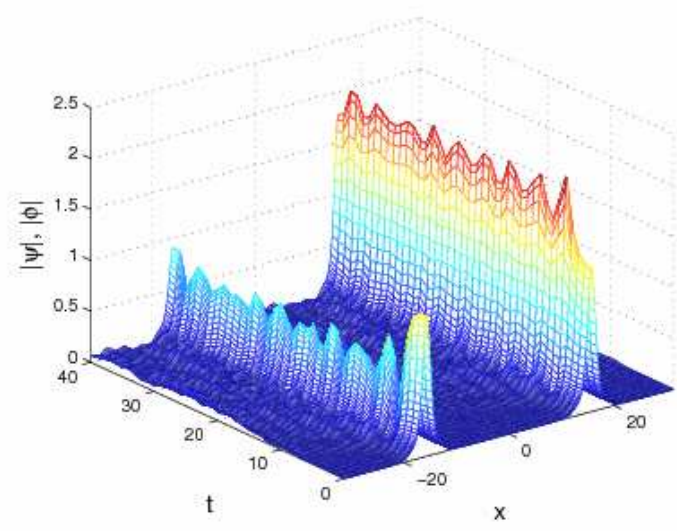

FIG. 6: (Color online) Perturbation-induced transformation of an unstable symmetric soliton into a stable asymmetric one in the attraction-attraction model with a relatively weak lattice $(\varepsilon=1, \kappa=1)$. The initial norm is $N=5.6$. Here and in Fig. 14 below, the two components of the solution are juxtaposed in the same panel.

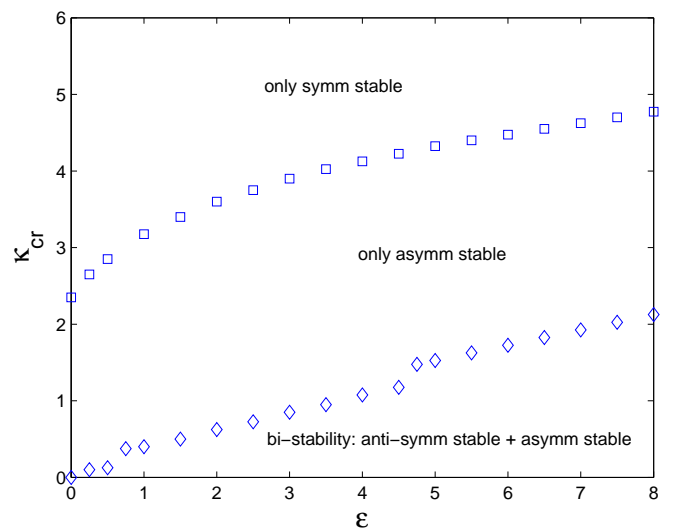

FIG. 7: (Color online) The attraction-attraction model: critical values of the coupling coefficient, $\kappa_{\mathrm{cr}}$, versus the lattice strength $\varepsilon$, for a fixed norm, $N=10$. The upper curve (squares) is, in fact, the

bifurcation line, i.e., the stability border of the symmetric solitons (which are stable above the border), and existence border of stable asymmetric solitons (they exist beneath the border). The lower curve

(rhombuses) is the stability border of antisymmetric solitons, which are stable beneath it, thus making the lower area a bistability region.

indeed, while, in weak lattices, they are completely destroyed by growing perturbations.

Results of the analysis are summarized in Fig. 7 in the form of a diagram showing the stability regions of the symmetric, antisymmetric, and asymmetric solitons in the $(\varepsilon, \kappa)$ plane. To this end, we define, in addition to the above-mentioned bifurcation value, $\kappa_{\text {bif }}$, which plays the role of the instability border of symmetric solitons (they are unstable, for given $\varepsilon$ and $N$, at $\kappa<\kappa_{\text {bif }}$, and stable at $\kappa \geq \kappa_{\text {bif }}$ ) and existence border of stable asymmetric solitons (they exist at $\kappa<\kappa_{\text {bif }}$ ), a critical value which separates stable and unstable antisymmetric solitons: they are stable at $\kappa \leq \kappa_{\mathrm{cr}}$, and unstable at $\kappa>\kappa_{\mathrm{cr}}$.

The stability analysis demonstrates that the branch of the antisymmetric solitons changes its character from unstable to stable as a whole, with the increase of $\varepsilon$ at fixed $\kappa$ [see Figs. 3(a) and (c)], or decrease of $\kappa$ at fixed $\varepsilon$. In other words, a situation has not been found when a part of the branch of antisymmetric solitons would be stable, while its other part is unstable. Note that the segment of the branch extending through the Bloch band, where the antisymmetric solitons are embedded ones, is stable too. 


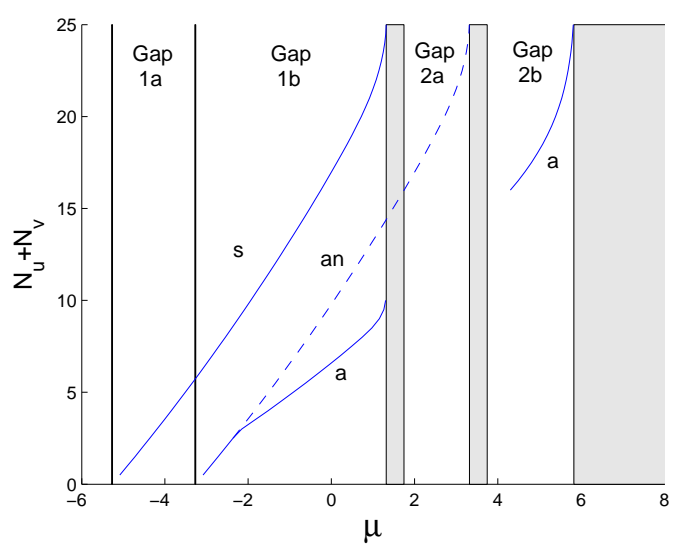

(a)

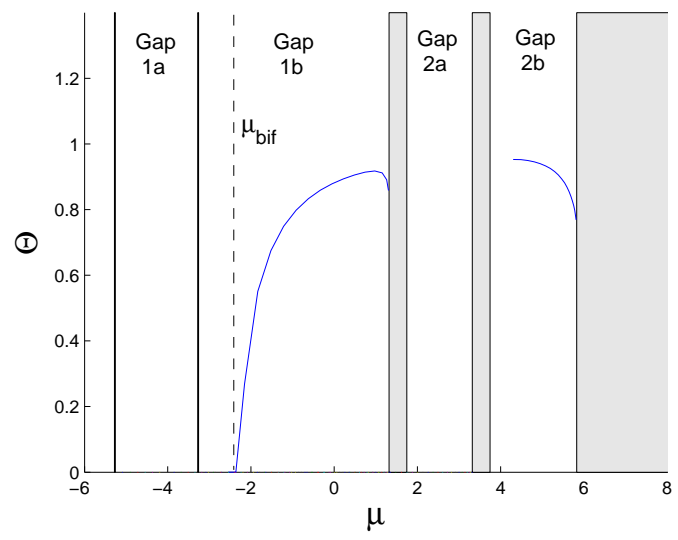

(b)

FIG. 8: (color online) Soliton families in the repulsion-repulsion model with $\varepsilon=8$ and $\kappa=1$. Panels (a) and (b) have the same meaning as in Fig. 3.

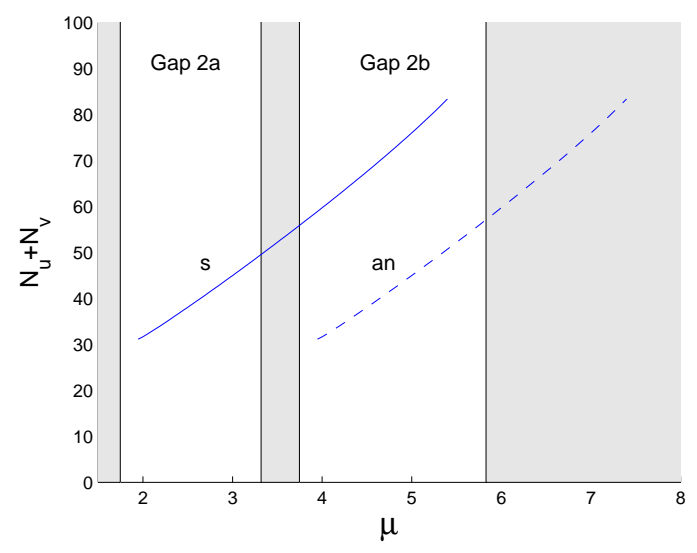

FIG. 9: (Color online) Continuation of Fig. 8 to larger values of the soliton's norm.

\section{B. The repulsion-repulsion system}

In the single-component GPE with repulsion, solitons may only be found in finite bandgaps, therefore they are called gap solitons [1, 3]. In the coupled system with repulsion in both cores, symmetric and antisymmetric states may be composed of single-component gap solitons as per Eqs. (7) and (8). Families of the solitons in the RR system, generated from the gap solitons belonging to the first and second finite bandgaps in the single-component GPE, are shown in Figs. 8 and 9, along with families of asymmetric solitons bifurcating from them. In particular, the symmetric branch in subgaps 2a and 2b in Fig. 9 (which extends across the Bloch band separating the two subgaps), and antisymmetric branch in subgap 2b (it extends into the adjacent Bloch band) may be regarded as continuations of the, respectively, symmetric branch in subgap $1 \mathrm{~b}$, and antisymmetric one in subgap 2a, which are displayed in Fig. 8. On the other hand, the origin of the stable branch of asymmetric solitons in subgap $2 \mathrm{~b}$ in Fig. 8 is not clear, as numerical problems impede to continue it in the direction of decreasing $\mu$ and $N$.

On the contrary to the situation in the AA model [cf. Figs. 3 (a) and (c)], in the present case the symmetric branch does not undergo any bifurcation, while asymmetric solitons bifurcate supercritically from the antisymmetric branch. Dependences of asymmetry ratio $\Theta$ on $\kappa$, and of the bifurcation value, $\kappa_{\text {bif }}$, on $N \equiv N_{u}+N_{v}$ for asymmetric solitons in the RR system are quite similar to those in its AA counterpart, which were shown above in Fig. 团 (therefore, the dependences for the RR system are not displayed here). 


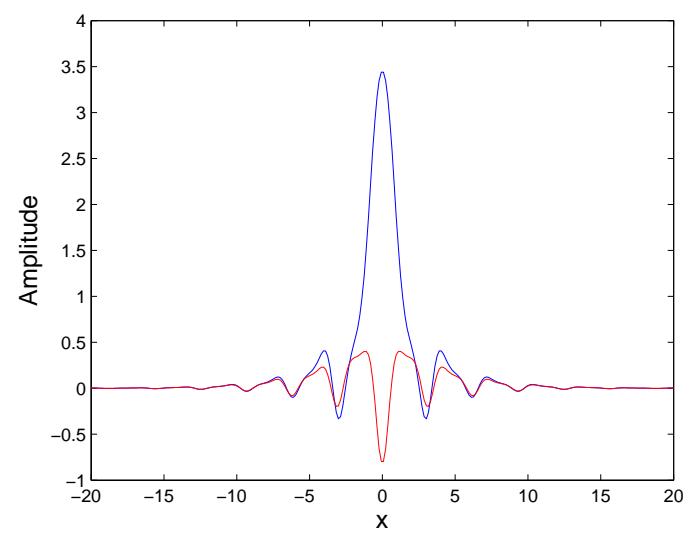

FIG. 10: (Color online) An asymmetric soliton in the repulsive-repulsive system, with $\epsilon=8, \kappa=1$, $N_{u}=19, N_{v}=0.9, \mu=5.39$.

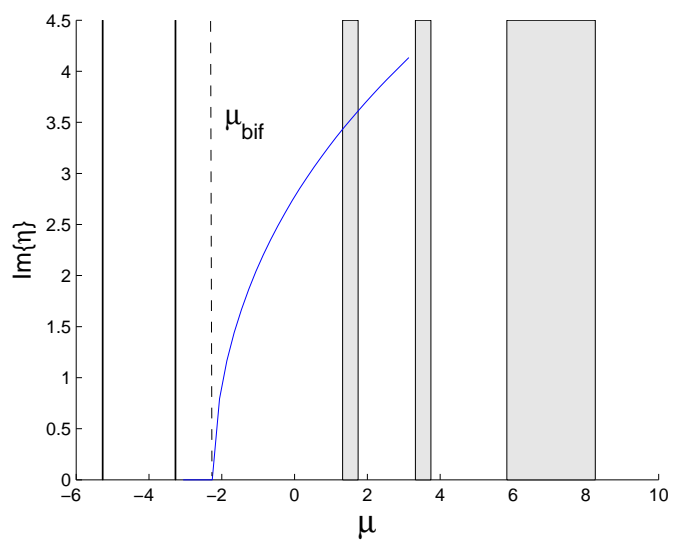

FIG. 11: (Color online) The instability growth rate for antisymmetric solitons in the first two (sub)gaps in the repulsion-repulsion model with $\kappa=1$ and $\varepsilon=8$.

A typical shape of the asymmetric soliton is presented in Fig. 10. It is worthy to note that both symmetric and antisymmetric soliton branches, but not asymmetric ones, may become embedded, crossing Bloch bands which separate the subgaps.

Similar to what was reported above for the AA model, the reduced eigenvalue problem based on Eqs. (14) and (15) was solved numerically to determine the stability of symmetric and antisymmetric gap solutions in the RR system (the VK criterion is irrelevant for self-defocusing models). The analysis demonstrates that the families of symmetric solitons presented in Figs. 8 and 9) are completely stable if the OL is strong enough, but symmetric solitons may be unstable in a weak lattice (for instance, at $\varepsilon=1$ ). This property is reminiscent of the AA system, where the antisymmetric solitons are unstable at $\varepsilon=1$, and stable at large $\varepsilon$, see Figs. 3 5and 7. Further, antisymmetric solitons are stable before the (anti)symmetry-breaking bifurcation, i.e., at $\mu \leq \mu_{\text {bif }}$, and, quite naturally, unstable at $\mu>\mu_{\text {bif }}$ (even if the antisymmetric branch does not seem to be continuously connected to the bifurcation point, see Fig. 91). In the latter case, the instability growth rate for the antisymmetric solutions is shown in Fig. 11.

Asymmetric solitons bifurcating from the antisymmetric ones are stable whenever they exist, which was checked by solving the full eigenvalue problem for them, based on Eqs. (11). As well as in the case of the AA system, these results imply bistability, provided that the OL is strong enough. Indeed, the symmetric solitons are then stable, and, along with them, either antisymmetric solitons or asymmetric ones (below or above the bifurcation, respectively) are stable too.

The predicted stability and instability of symmetric, antisymmetric, and asymmetric solitons has been 


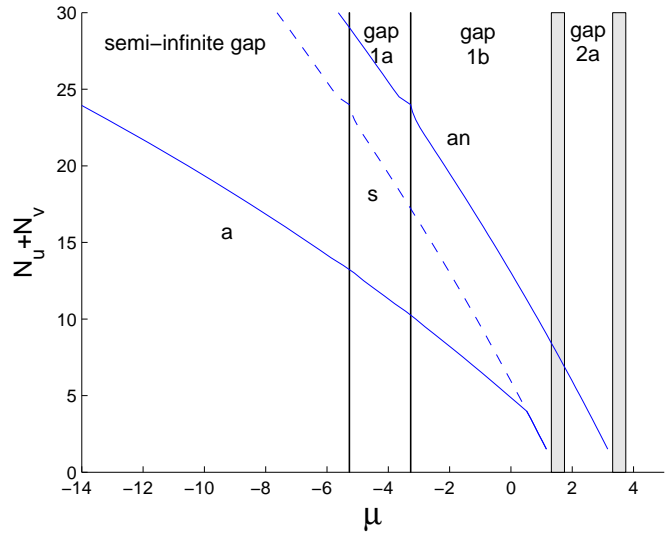

(a)

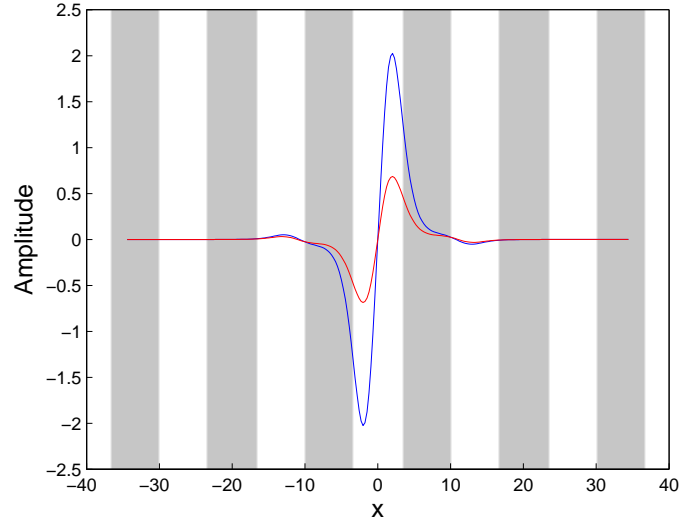

(b)

FIG. 12: (Color online) (a) Families of twisted (odd) solitons in the attraction-attraction system, with $\varepsilon=8$ and $\kappa=1$. Symmetric twisted solitons are stable up to the bifurcation point, beyond which they are unstable, while the emerging twisted asymmetric solitons are stable. Antisymmetric twisted solitons are stable everywhere (actually, because the lattice is strong enough), cf. Fig. 3(c). (b) An example of an asymmetric twisted soliton with $N_{u}=5.3, N_{v}=0.7, \mu \approx-0.66$. In this figure and in some figures below, shaded vertical stripes depict the lattice potential which supports the solitons.

verified by direct simulations too. In particular, the antisymmetric solitons, if unstable, tend to rearrange themselves into their stable asymmetric counterparts.

\section{Twisted solitons and bound states}

Twisted (alias odd) solitons, which feature two out-of-phase amplitude peaks in one period of the underlying lattice, were found and shown to be stable in the single-component GPE with attractive nonlinearity [26] (unlike formally similar subfundamental gap solitons in the model with self-repulsion, which are unstable 27]).

We have studied spontaneous symmetry breaking of twisted solitons in the AA system. Families of twisted solitons in this system are shown in Fig. 12(a). The behavior is generally similar to that of the fundamental solitons in the same (AA) model: a stable asymmetric branch bifurcates from the symmetric one, leaving it unstable; however, it is worthy to note that, in the case shown in Fig. 12(a), the bifurcation occurs in the finite (sub)gap, 1b, while the similar bifurcation of fundamental solitons was observed, in Figs. 3(a) and (c), in the semi-infinite gap. The entire antisymmetric branch of the twisted solitons is stable, provided that the lattice is strong enough, or unstable otherwise. A typical example of the asymmetric twisted soliton is shown in Fig. 12(b).

Bound states of $\pi$-out-of-phase fundamental solitons, which also feature the odd parity, but include peaks separated by an empty lattice site, have been found too, in the AA and RR systems alike (in the singlecomponent GPE with the repulsive nonlinearity, the presence of an empty site between the peaks is a necessary condition for the existence of a stable bound state of two out-of-phase fundamental gap solitons 27, 29]). Families of the bound states of this type are shown in Fig. [13, being quite similar to fundamentalsoliton families, cf. Figs. 3 and 8 A difference is observed in the evolution of symmetric and anti-symmetric solitons destabilized by the bifurcation: rather than rearranging into asymmetric solitons, they give rise to persistent breathers, as shown in Fig. 14.

\section{ASYMMETRIC SYSTEMS}

As said in Introduction, the two-core system can be made asymmetric by either assuming that the signs of the scattering lengths are opposite in the parallel-coupled traps, or by admitting a mismatch between the 


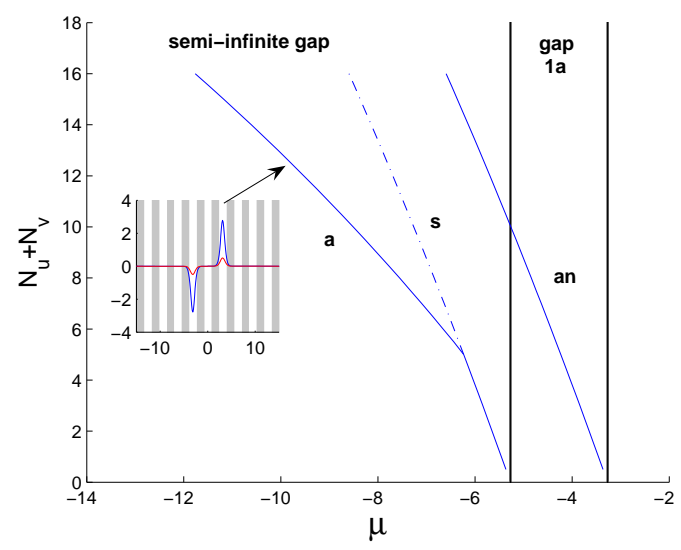

(a)

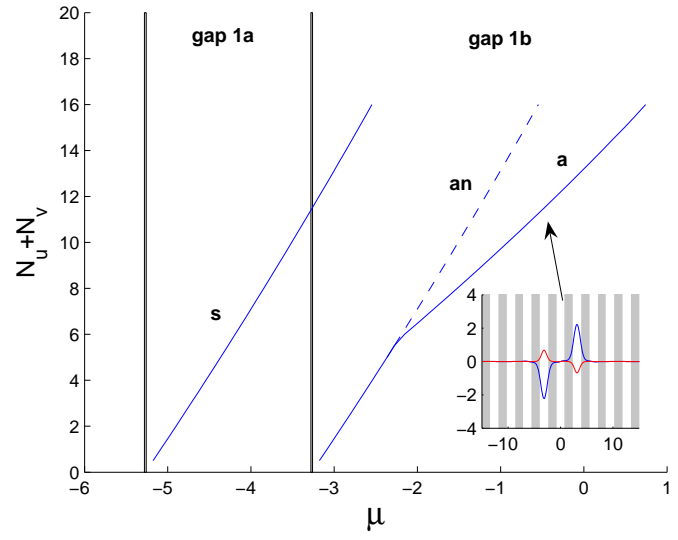

(b)

FIG. 13: (Color online) Families of twisted bound states, for $\varepsilon=8, \kappa=1$. Insets show typical bound-soliton profiles. Panels (a) and (b) pertain to the attraction-attraction and repulsion-repulsion system, respectively.

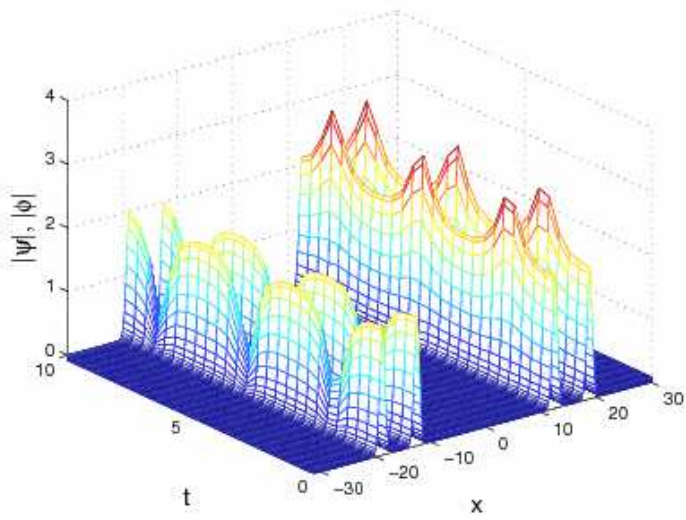

(a)

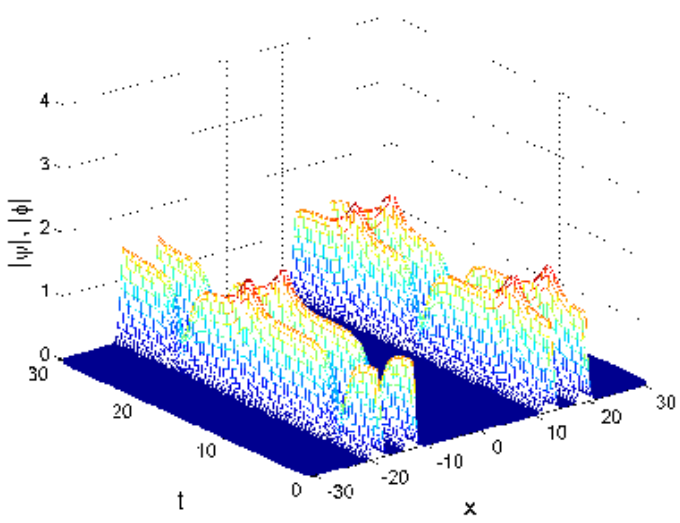

(b)

FIG. 14: (Color online) Evolution of unstable twisted (odd) bound states of fundamental solitons in the system with $\varepsilon=8, \kappa=1$. (a) A bound state of symmetric solitons in the attraction-attraction system; (b) a bound state of antisymmetric solitons in the repulsion-repulsion system. In either case, formation of a long-lived breather is observed.

two OLs. Both cases are considered in this section.

\section{A. The repulsion-attraction system with aligned lattices}

In the RA system (we set $g_{1}=-1, g_{2}=1$, assuming opposite signs but equal absolute values of the nonlinear coefficients in the components), numerical solution of Eqs. (6) reveals two soliton families, viz., ones with a dominant repulsive component $\left(N_{u}>N_{v}\right)$ residing in subgap 1b, or with a dominant attractive component $\left(N_{u}<N_{v}\right)$ in the semi-infinite bandgap (solitons in two-core RA systems, but without the lattice, were considered in Refs. [30]). Generic examples of such families are presented in Fig. 15, and typical examples of the respective soliton shapes are displayed in Fig. 16

The computation of the eigenvalues for perturbation modes demonstrates that both families are completely stable. Note that the stability of the attraction-dominated solutions, with $N_{u}<N_{v}$, is also suggested by 


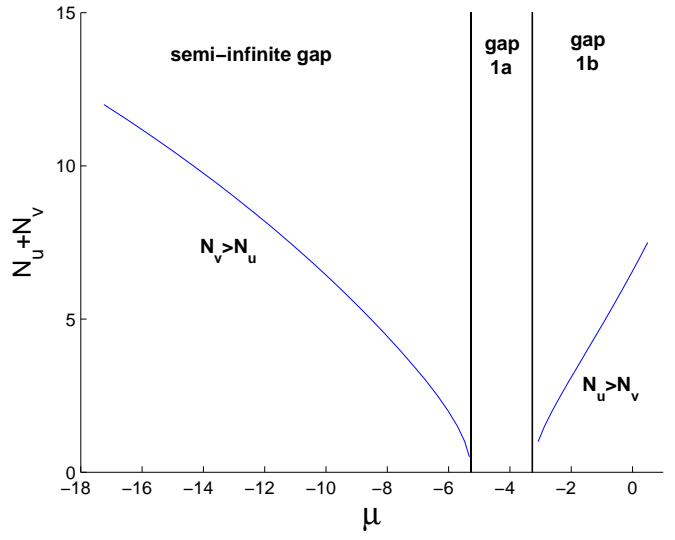

(a)

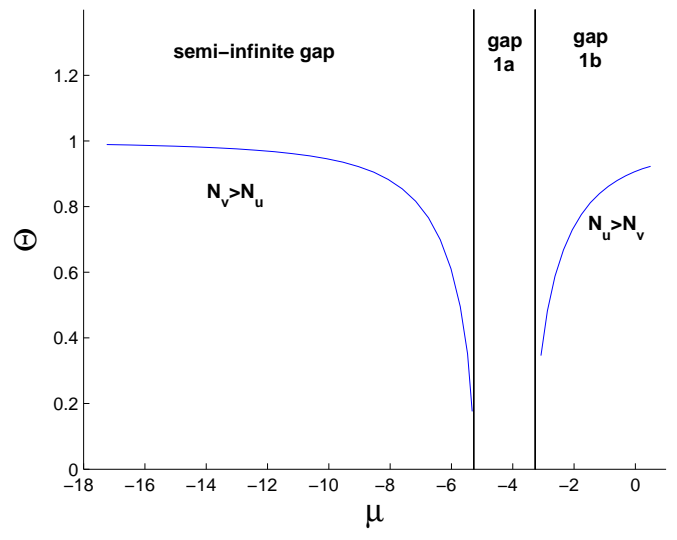

(b)

FIG. 15: (Color online) Solitons families in the repulsion-attraction model $\left(-g_{1}=g_{2}=1\right)$ with $\varepsilon=8$ and $\kappa=1$.

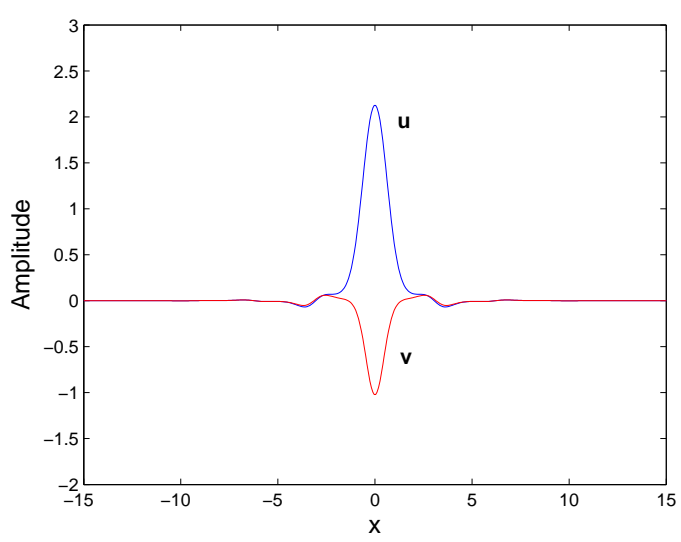

(a)

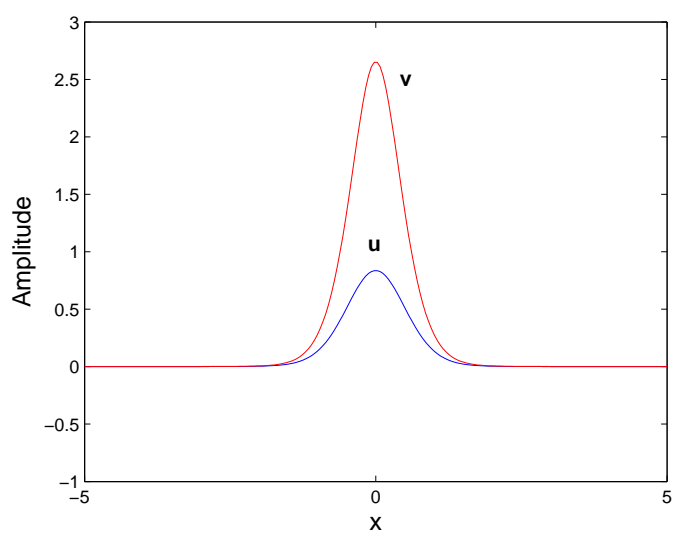

(b)

FIG. 16: (Color online) Examples of solitons in the repulsion-attraction model, with $-g_{1}=g_{2}=1$, and $\varepsilon=8, \kappa=2$. (a) $N_{u}=5.1, N_{v}=0.9, \mu \approx-0.105$; (b) $N_{u}=0.6, N_{v}=5.4, \mu \approx-9.58$. In either case, the total norm is $N=6$.

the VK criterion, if applied to dependence $N(\mu)$ in Fig. 15)(a) (for the repulsion-dominated solutions, with $N_{u}>N_{v}$, the VK criterion is irrelevant, as mentioned above).

\section{B. Bifurcations and pseudo-bifurcations in systems with mismatched lattices}

To consider effects of the mismatch between the OLs in the two cores, we will here focus on Eqs. (11) with $\Delta=\pi / 2$ and $\Delta=\pi$ (the latter value corresponding to the largest mismatch, similar to a system of parallel Bragg gratings with the maximum mismatch, that was recently considered in Ref. 31]). For all types of the nonlinear interactions in the cores, at $\kappa=0$ the solitons in the decoupled shifted lattices were taken as $u(x)=\hat{u}_{0}(x), v(x)=0$, where $\hat{u}_{0}(x)$ is the corresponding soliton in the single-component GPE. At this point, the asymmetry ratio defined in Eq. (9) is at its maximum, $\Theta=1$. As the linear-coupling coefficient, $\kappa$, increases, the solution becomes less asymmetric.

Figure 17 displays soliton families found in the AA system. As in Ref. [32], which treated a mismatched system of parallel-coupled Bragg gratings, we distinguish between asymmetric and quasi-symmetric (QS) 


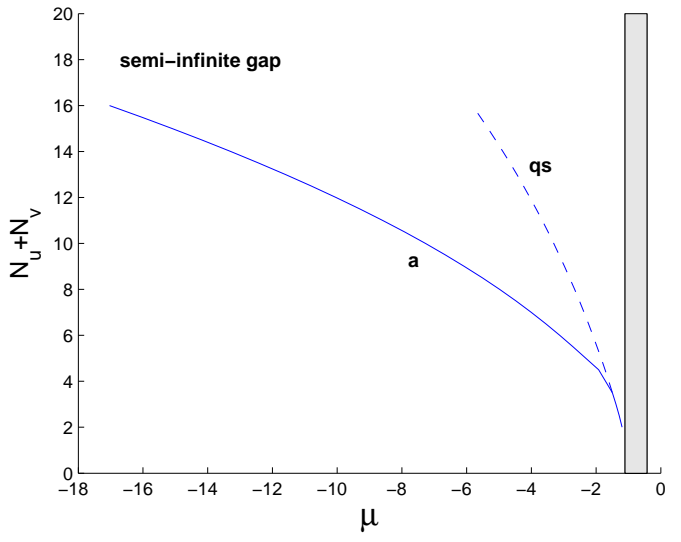

(a)

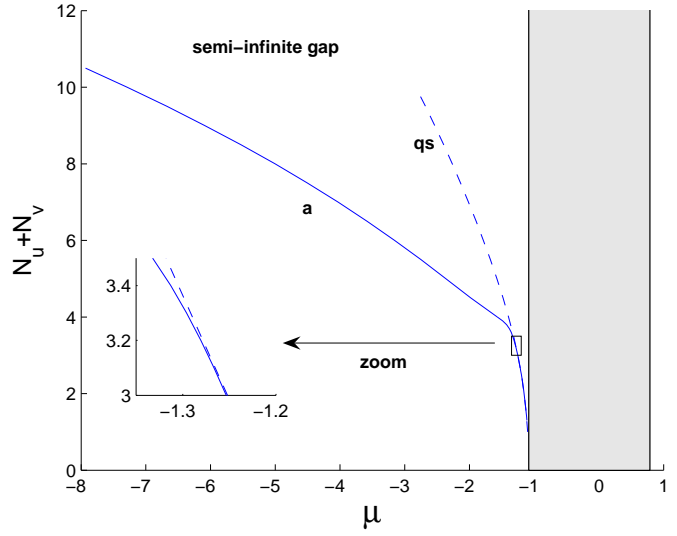

(b)

FIG. 17: (Color online) Solitons families in the attraction-attraction system with mismatched lattices for $\varepsilon=1$ and $\Delta=\pi / 2$ (a) and $\Delta=\pi(\mathrm{b})$. Labels "a" and "qs" refer to asymmetric and quasi-symmetric branches, respectively.

solitons [a branch of quasi-antisymmetric (QAS) solutions was found too but is not shown, as it is completely unstable, cf. Fig. 3. QS solitons have equal norms in both components, i.e., $\Theta=0$, and feature similar, although not identical, profiles of the components. The bifurcation chart for the AA system with $\Delta=\pi / 2$ is very similar to the one for the same system with aligned OLs $(\Delta=0)$, the symmetric solutions being replaced by their QS counterparts. In particular, the asymmetric branch bifurcates from the QS one.

At first glance, it may seem that, in the AA system with $\Delta=\pi$, the branch of asymmetric solitons also bifurcates from the QS one. However, this is not the case. A blow-up (inset in Fig. 17b) shows a pseudo-bifurcation, which means that asymmetric and QS branches gradually approach each other to a point where they seem indistinguishable, but they never merge, therefore the QS solutions always remain (strictly speaking) unstable, while the asymmetric ones are always stable (the stability is discussed in more detail below). In Fig 18, we display an example of comparison between shapes of asymmetric and QS solitons with equal norms, $N=3.5$, when they are very close to each other, the asymmetric one having $\Theta \approx 10^{-4}$ (its QS counterpart has $\Theta$ exactly equal to zero). Note that the centers of the two components in the QS soliton are slightly separated, while in the asymmetric soliton they exactly coincide.

Figure 19 additionally illustrates the difference between the true quasi-symmetry-breaking bifurcation in the AA system with $\Delta=\pi / 2$, and the pseudo-bifurcation in the system with $\Delta=\pi$. In the latter case, the branches closely approach but never merge, cf. Fig. 17

Typical shapes of strongly asymmetric solitons in the mismatched AA model are displayed in Fig. 20. Naturally, the solitons are more asymmetric at $\Delta=\pi$.

Families of solitons found in the mismatched RR system are presented in Fig. 21] The QS branch is called this way only in the sense that peak amplitudes of the two components have the same sign. In fact, profiles of the components in the QS soliton may be very different, as insets show in Fig. 21. The quasiantisymmetry-breaking bifurcation in the RR system and respective pseudo-bifurcation are illustrated by Fig. 22, and a set of typical profiles of the solitons is displayed in Fig. 23.

Stability of the solitons in the mismatched AA and RR systems was studied in direct simulations of Eqs. (11) over a wide range of values of $N$ and $\kappa$. In either case (as in the aligned system), the asymmetric solitons were found to be stable whenever they exist, and the branch which gives rise to the bifurcation is stable before the bifurcation and unstable afterwards. Further, QAS and QS solitons in the AA and RR system, respectively, are unstable at small $\varepsilon$ and stable at larger $\varepsilon$. When QS solitons are unstable in the AA system, they evolve into their stable asymmetric counterparts. Similarly, in the misaligned RR system, QAS solitons which were destabilized by the bifurcation (or which are unstable due to the pseudo-bifurcation) transform themselves into stable asymmetric solutions.

Soliton families in the mismatched system of the RA type were investigated too. It was found that the corresponding picture is quite similar to that presented for the RA system without mismatch in Fig. 15. 


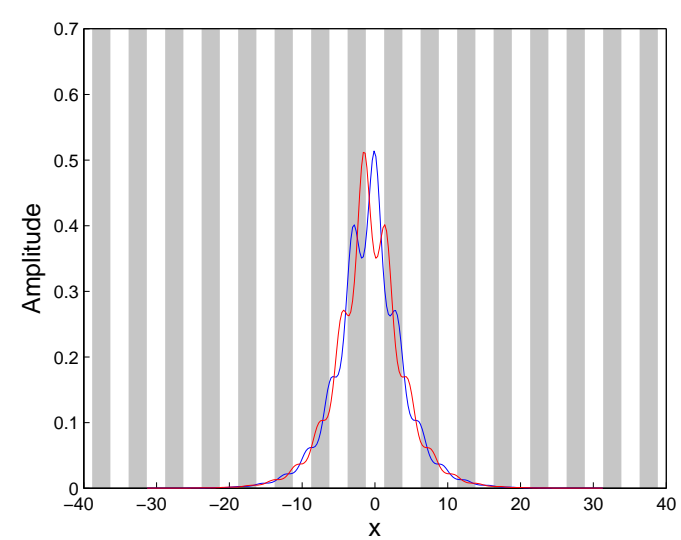

(a)

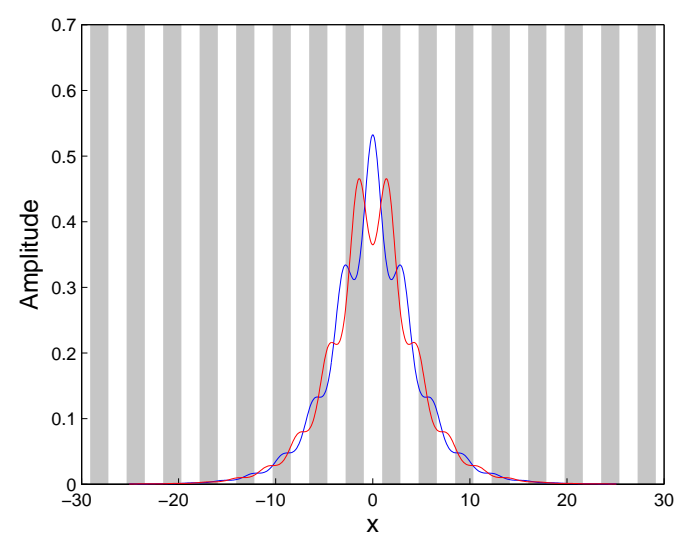

(b)

FIG. 18: (Color online) Comparison of soliton profiles in the attraction-attraction model with $\Delta=\pi$ and $\varepsilon=1, \kappa=1$. (a) A quasi-symmetric soliton, with $\Theta=0$; (b) a slightly asymmetric soliton, with $\Theta \approx 10^{-4}$. Both solitons have equal norms, $N=3.5$. Here and below, the set of vertical shaded stripes represents the periodic lattice potential in the first core.

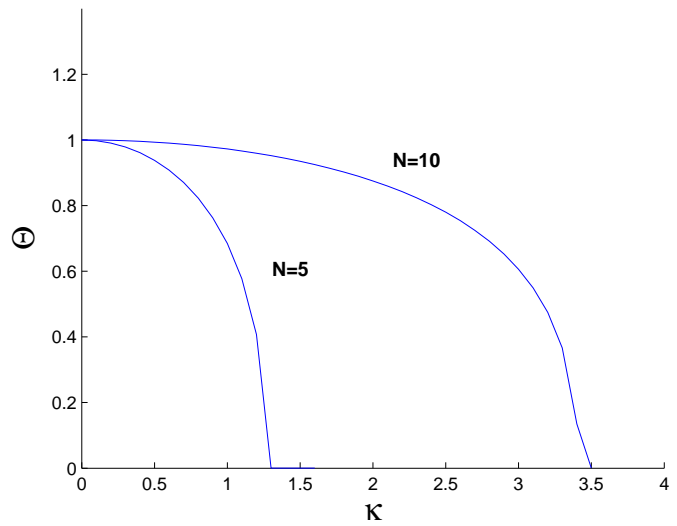

(a)

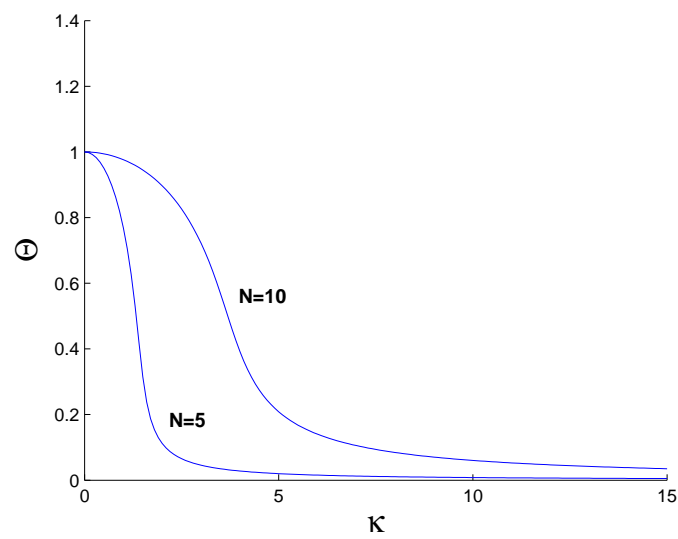

(b)

FIG. 19: (Color online) The symmetry ratio for solitons in the mismatched attraction-attraction model with $\varepsilon=1$ versus the coupling coefficient, $\kappa$ : (a) $\Delta=\pi / 2$; (b) $\Delta=\pi$ (similar dependences for the aligned system, with $\Delta=0$, are displayed in Fig. (4).

\section{BINARY MIXTURES WITH LINEAR COUPLING}

In this section we consider the model of a binary BEC in the single-core trap equipped with an OL. As explained in Introduction, the two components of the mixture $(\psi$ and $\phi)$ represent two different spin states of the same atom, and the linear coupling between them is induced by a spin-flipping electromagnetic wave. The corresponding system of the normalized GPEs for wave functions $\psi$ and $\phi$ takes the form

$$
\begin{aligned}
i \psi_{t}+\psi_{x x}+\varepsilon \cos (2 x) \psi+\left(g_{1}|\psi|^{2}+g_{12}|\phi|^{2}\right) \psi+\kappa \phi & =0, \\
i \phi_{t}+\phi_{x x}+\varepsilon \cos (2 x) \phi+\left(g_{2}|\phi|^{2}+g_{12}|\psi|^{2}\right) \phi+\kappa \psi & =0
\end{aligned}
$$

(in the single trap, there may be no mismatch between the lattice-potential terms in the two equations). As the interaction between atoms may be both attractive and repulsive, in this section we consider two basic cases: when all the three nonlinear terms are attractive, i.e., $g_{1}=g_{2} \equiv 1, g_{12} \geq 0$ (AAA), and when they all are repulsive, with $g_{1}=g_{2}=-1, g_{12} \leq 0(\mathrm{RRR})$. 


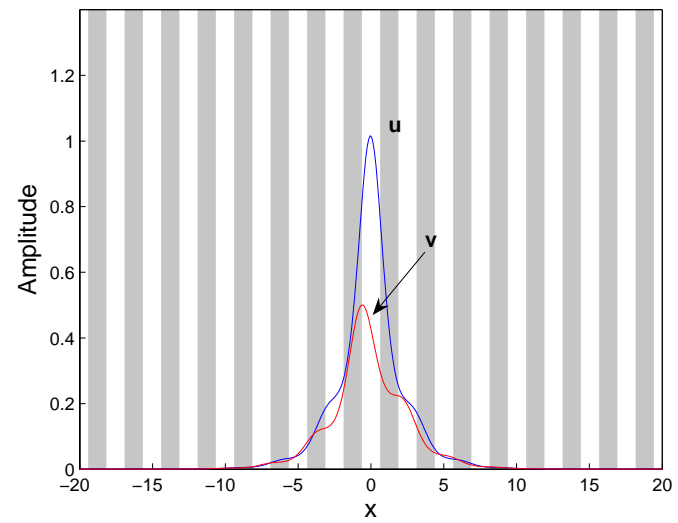

(a)

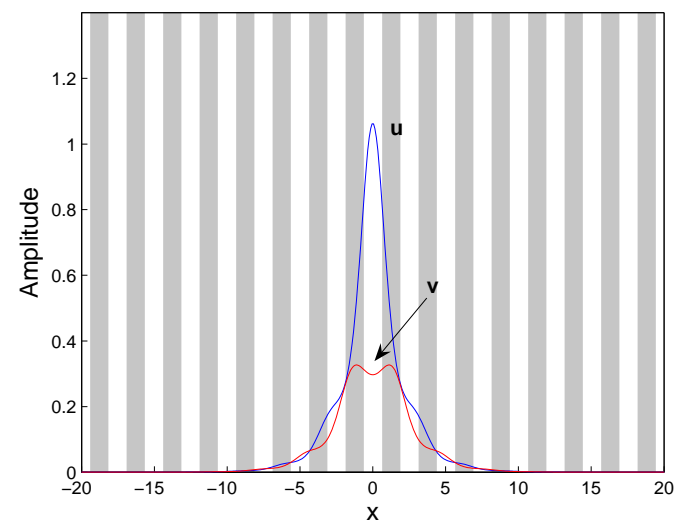

(b)

FIG. 20: (Color online) Typical asymmetric solitons in the mismatched attraction-attraction system with $\varepsilon=1:$ (a) $\Delta=\pi / 2, N=2.5, \Theta=0.51, \mu=-0.83, \kappa=0.4$; (b) $\Delta=\pi, N=2.5, \Theta=0.63, \mu=-0.85$,

$$
\kappa=0.4 \text {. }
$$

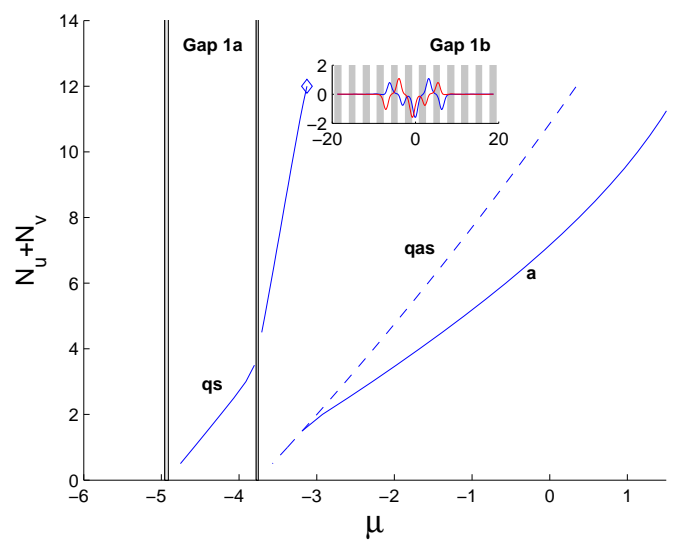

(a)

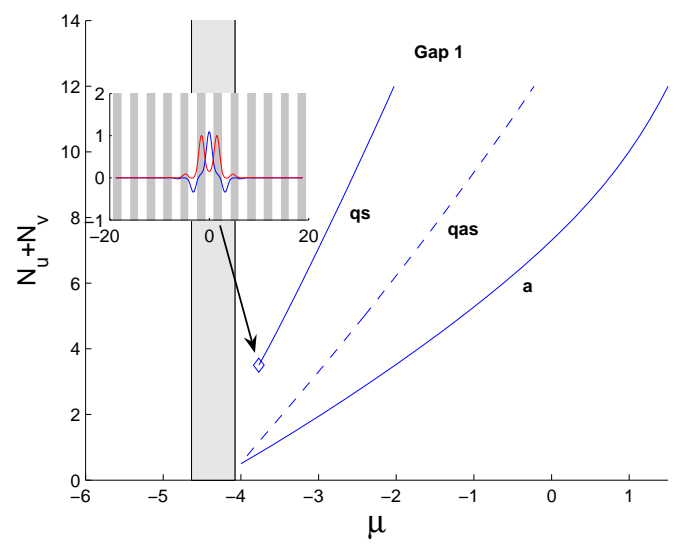

(b)

FIG. 21: (Color online) Soliton families in the misaligned repulsion-repulsion system, with $\varepsilon=8$. Labels "a", "qs", and "qas" refer to asymmetric, quasi-symmetric and quasi-antisymmetric branches, respectively. Insets show soliton profiles at points marked by diamonds. (a) $\Delta=\pi / 2$; (b) $\Delta=\pi$.

Bifurcation diagrams for the AAA and RRR systems are shown in Fig. 24. In the former case (AAA), stable asymmetric solitons bifurcate, as before, from symmetric ones if $0 \leq g_{12}<1$. However, if the inter-species nonlinear interactions dominate, i.e., for $g_{12}>1$, the character of the bifurcation changes, and asymmetric solitons bifurcate from antisymmetric ones. In the RRR system, a similar transition is observed: the asymmetric solitons are generated, as above, by the bifurcation from antisymmetric solitons if $0 \leq-g_{12}<1$, but, for $-g_{12}>1$, antisymmetric solitons bifurcate from symmetric ones. In the Manakov's case, $\left|g_{12}\right|=1$ [33], no bifurcation was found. Note that linearly coupled equations (17) without the lattice potential $(\varepsilon=0)$, but with the linear coupling present, $\kappa \neq 0$, are equivalent to the Manakov's integrable system with $\kappa=0$ [34], therefore the absence of the bifurcation in this case is not surprising.

Direct simulations of the AAA system demonstrate that the asymmetric solitons, whenever they exist, are stable, and, as usual, the bifurcation destabilizes the solitons from which the asymmetric ones emerge. In the RRR system, the stability situation is more complex. While asymmetric solitons bifurcating from the symmetric ones in the RRR system, i.e., in the case of $\left|g_{12}\right|>1$, are always stable, the asymmetric solitons bifurcating, in the same system, from the antisymmetric branch (at $0 \leq\left|g_{12}\right|<1$ ) may be both stable and 


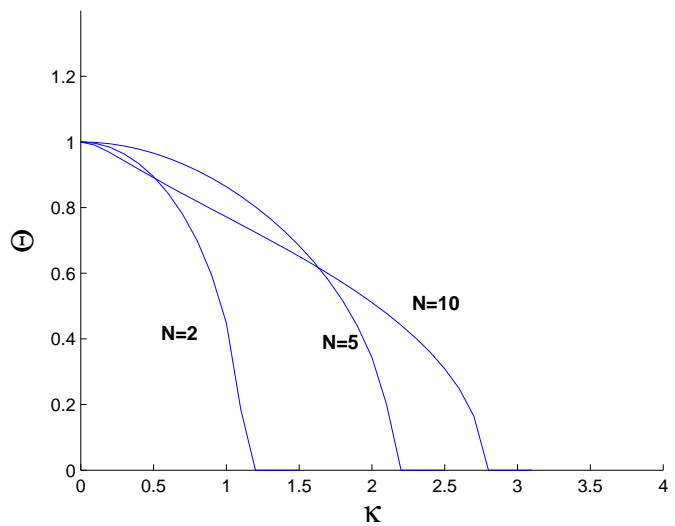

(a)

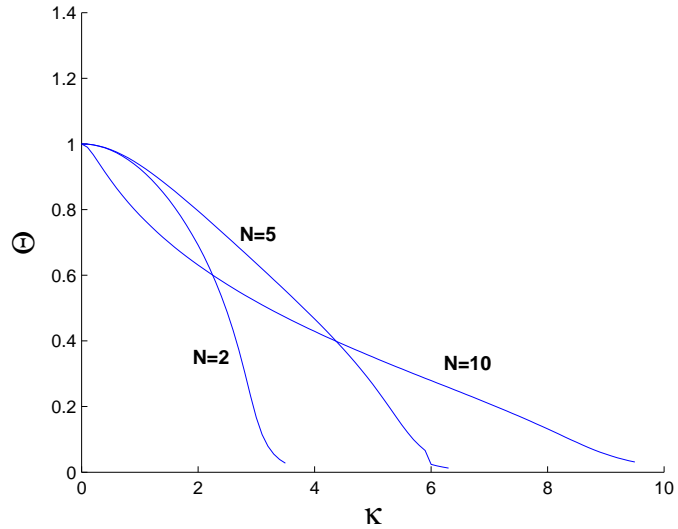

(b)

FIG. 22: (Color online) The asymmetry ratio of solitons in the mismatched repulsion-repulsion system with $\varepsilon=8:$ (a) $\Delta=\pi / 2$; (b) $\Delta=\pi$.

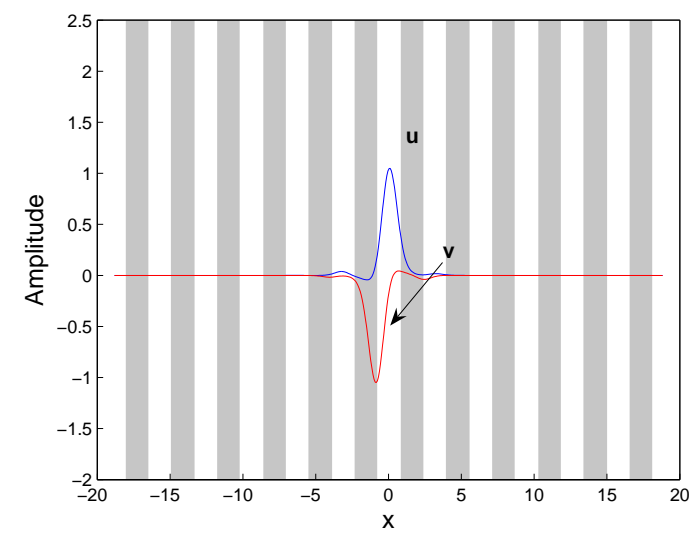

(a)

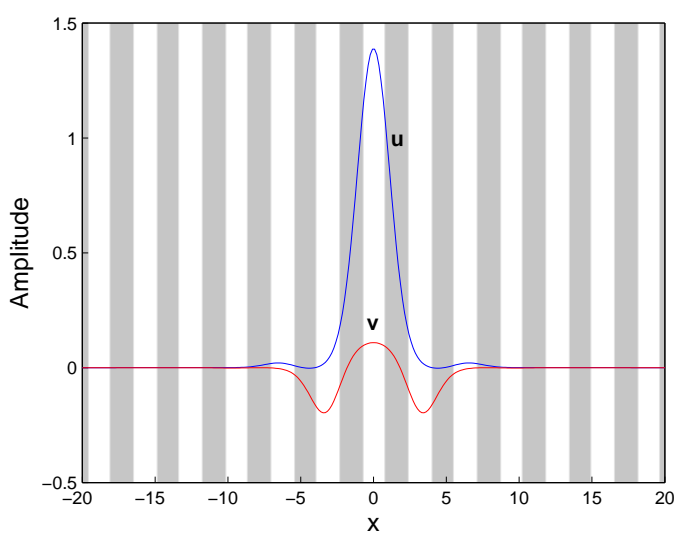

(c)

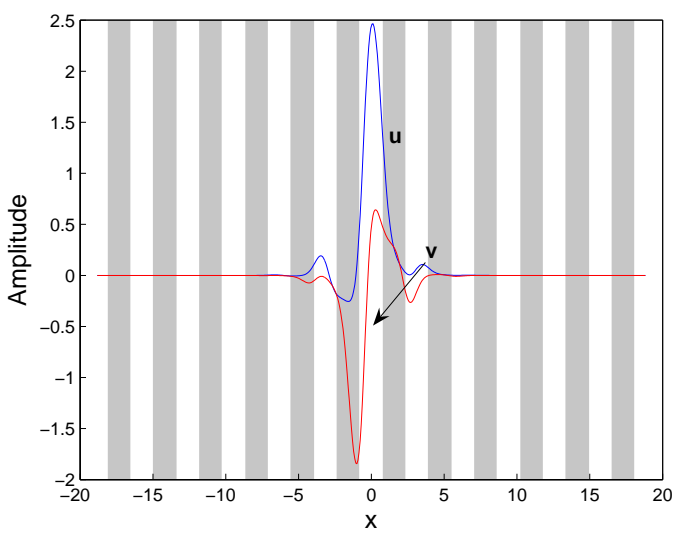

(b)

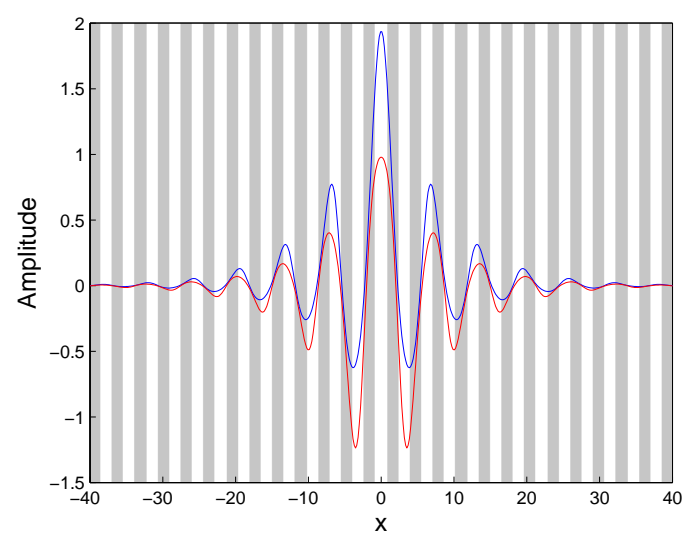

(d)

FIG. 23: (Color online) Profiles of asymmetric solitons in the mismatched repulsion-repulsion model with $\varepsilon=8$. (a) $\Delta=\pi / 2, N=2, \Theta=0, \mu=-2.81, \kappa=1.5$; (b) $\Delta=\pi / 2, N=10, \Theta=0.31, \mu=0.23, \kappa=2.5$; (c) $\Delta=\pi, N=2, \Theta=0.92, \mu=-2.96, \kappa=1$; (d) $\Delta=\pi, N=10, \Theta=0.13, \mu=-5.57, \kappa=8$. 


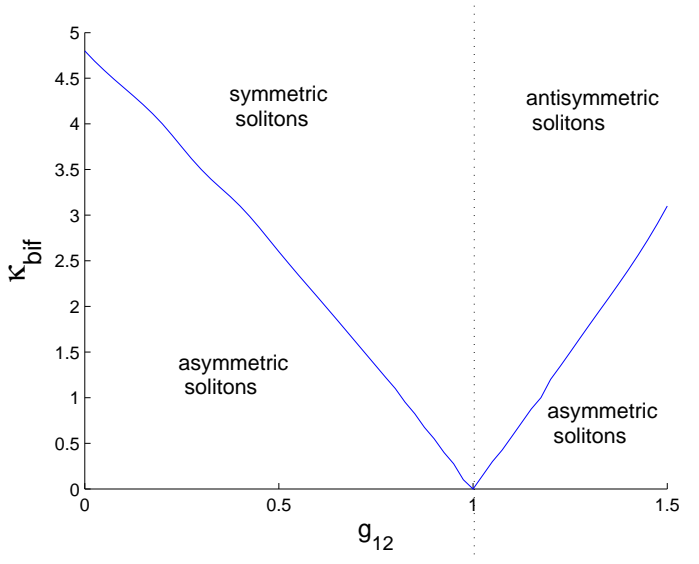

(a)

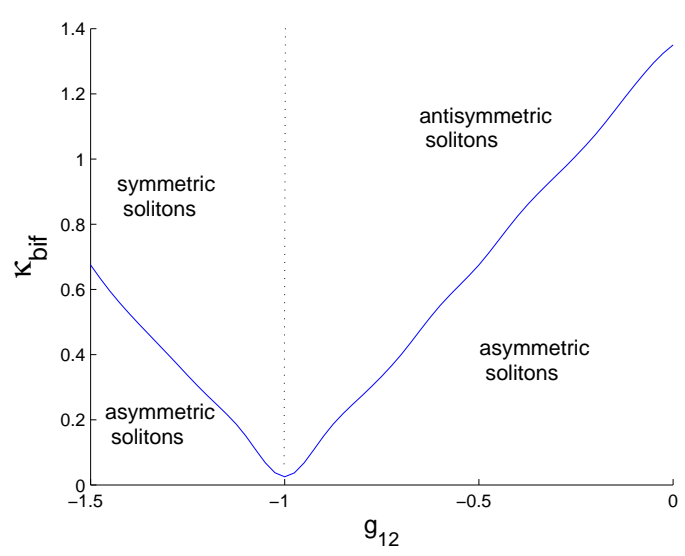

(b)

FIG. 24: The bifurcation value of the coupling coefficient, $\kappa_{\text {bif }}$, versus the relative strength of the interspecies nonlinear interaction, $g_{12}$, in the model of the binary mixture loaded into the optical lattice with strength $\varepsilon=8$ [Eqs. (17)]. The vertical dashed line divides regions in which asymmetric solitons bifurcate from symmetric and antisymmetric ones. (a) The model with attractive nonlinearities (for total norm $N=10$ ). In this system, the asymmetric solitons are always stable. (b) The model with repulsive nonlinearities (for $N=4$ ). In this case, all asymmetric solitons bifurcating from the symmetric ones are stable, while the solitons bifurcating from the antisymmetric branch are unstable if $\left|g_{12}\right|$ is close to 1 (but they are stable if $\left|g_{12}\right|$ is small).

unstable. We did not try to identify a border between the stable and unstable asymmetric solitons in the latter case in an accurate form; however, the asymmetric solitons in the RRR system are definitely stable for $\left|g_{12}\right| \rightarrow 0$ (which complies with the results reported above for the RR system with $g_{12}=0$ ), and they are unstable for $\left|g_{12}\right|$ close to (but smaller than) 1. In that case, direct simulations demonstrate that unstable asymmetric solitons transform into persistent breathers (not shown here). We did not systematically study the stability of the nonbifurcating families, i.e., antisymmetric and symmetric ones in the AAA and RRR systems, respectively.

\section{CONCLUSION}

In this work, we have studied families of soliton states in a symmetric set of two effectively one-dimensional traps (cores) equipped with OLs (optical lattices), filled with self-attractive or self-repulsive BEC, and coupled by linear tunneling (the same model may be also realized in terms of spatial solitons in two parallel planar optical waveguides, which carry lattices in the form of a transverse modulation of the refractive index). Asymmetric systems, with a phase shift $\Delta$ between the two OLs, as well as with opposite signs of the nonlinearity in the BEC confined in the different traps, were considered too. In the spectrum of the dual-core system, the linear coupling between the cores splits finite bandgaps induced by the single OL into subgaps, or partly closes them. Influence of mismatch $\Delta$ on the spectrum was considered too, with a conclusion that large $\Delta$ tends to make the spectrum qualitatively similar to that in the single-core model. In the full nonlinear model, families of symmetric and antisymmetric solitons, as well as asymmetric solitons generated by symmetry-breaking bifurcations, have been constructed. This was done in the case of attraction or repulsion in both cores (AA and RR systems), as well as in the mixed (RA) system. Deformation of the soliton families in the AA and RR system under the action of mismatch $\Delta$ between the two OLs was investigated too.

In the AA and RR systems, asymmetric solitons bifurcate supercritically from symmetric and antisymmetric ones, respectively. In the former case, symmetric and asymmetric fundamental solitons were found only in the semi-infinite gap, while antisymmetric solitons were also discovered in the first finite subgap, as well in the Bloch band separating it from the semi-infinite band (in the latter case, the antisymmetric solitons have the character of embedded ones). In the RR system, solitons are located in finite bandgaps, 
and, additionally, antisymmetric and symmetric solitons were found as embedded ones inside Bloch bands. In both systems (AA and RR), the asymmetric solitons are always stable, while the branch which gives rise to them is stable before the bifurcation, and unstable afterwards. The nonbifurcating branches, i.e., antisymmetric and symmetric solutions in the AA and RR systems, respectively, are unstable or stable as a whole in weak and strong OL, respectively. The corresponding stability region was identified in a complete form for the AA system, in the parameter plane of coupling coefficient $\kappa$ and OL strength $\varepsilon$. In the systems of both AA and RR types, bistability regions were found, in which the stable asymmetric solitons coexist with either antisymmetric or symmetric ones, respectively. If the symmetric soliton in the AA system, or the antisymmetric soliton in the RR system was destabilized by the bifurcation, its evolution tends to transform it into a stable asymmetric soliton, in either system. In the RA system, two soliton families were found, dominated by either the self-attractive or repulsive component, both being entirely stable.

In the AA system, we have also studied families of twisted (odd) solitons, featuring two out-of-phase peaks in a cell of the OL. The behavior of these families is, generally, similar to that of the fundamental solitons, including the bifurcation of the symmetric branch into an asymmetric one, and bistability; however, unlike the situation with the fundamental solitons, the symmetry-breaking bifurcation of the twisted solitons occurs in a finite bandgap. In addition, $\pi$-out-of-phase bound states of fundamental solitons were constructed in both the AA and RR systems. Their behavior too is quite similar to that of the respective fundamental solitons, with a difference that, past the bifurcation, an unstable bound state tends to arrange itself into a persistent breather (rather than into a stationary bound state of the asymmetric type).

The study of the systems with the mismatch, $\Delta$, between the OLs in the two cores demonstrates that former symmetric and antisymmetric solitons turn into QS (quasi-symmetric) and QAS (quasi-antisymmetric) ones. For $\Delta=\pi / 2$, the deformation is mild, and the behavior of various soliton families remains qualitatively the same as in the system with $\Delta=0$; in particular, well-defined bifurcations which generate asymmetric solitons from QS or QAS ones, in the mismatched AA and RR system, respectively, occur despite the presence of the mismatch (in a system of mismatched parallel-coupled Bragg gratings, similar observations were recently made in Ref. [32]). However, when the mismatch attains its maximum, $\Delta=\pi$, the situation becomes different: instead of the bifurcations, both the AA and RR systems demonstrate pseudo-bifurcations, when the branch of asymmetric solutions does not really bifurcate from that of QS or QAS solutions, but rather goes very close and only asymptotically merges into it. Thus, the asymmetric branch always exists and is stable in the latter case, while its QS or QAS counterpart is always unstable (although the instability is extremely weak when it approaches the stable asymmetric branch). To the best of our knowledge, the present work reports the first example of the pseudo-bifurcation in a mismatched binary system (in a system of mismatched Bragg gratings, no such effect was observed [32]).

Finally, we have considered a model of the single-core trap, equipped with the OL and filled with a binary mixture, assuming both the nonlinear interaction between the two species, and linear coupling between them, induced by a resonant spin-flipping field, if the species correspond to different spin states of the same atom. In this case, we have found that the bifurcations generating asymmetric solitons from symmetric and antisymmetric ones are qualitatively similar to their counterparts in the model without the nonlinear interaction between the species, if this interaction is weaker than the intra-species nonlinearity; otherwise, the bifurcations switch their character, so that the asymmetric solitons emerge from antisymmetric and symmetric ones in the AA and RR systems, respectively.

A very natural extension would be to consider a two-dimensional version of the models introduced in this work, which may also directly apply to BEC in a dual-core pancake-shaped trap, or to a binary mixture in a single-core nearly flat trap. The work in this direction is currently in progress, and results will be reported elsewhere. It may also be possible to consider the spontaneous symmetry breaking in a degenerate fermion gas filling a dual-core trap, using a relatively simple description of the fermion gas based on the Thomas-Fermi (alias mean-field-hydrodynamic) approximation, which was developed, for various settings, in Refs. [35]. 


\section{Acknowledgement}

This work was supported, in a part by the Israel Science Foundation through the Center-of-Excellence grant No. 8006/03.

[1] O. Morsch and M. Oberthaler, Rev. Mod. Phys. 78, 179 (2006).

[2] F. K. Abdullaev, A. Gammal, A. M. Kamchatnov, and L. Tomio, Int. J. Mod. Phys. B 19, 3415-3473 (2005).

[3] F. Kh. Abdullaev, B. B. Baizakov, S. A. Darmanyan, V. V. Konotop, and M. Salerno, Phys. Rev. A 64, 043606 (2001); I. Carusotto, D. Embriaco, and G. C. La Rocca, ibid. 65, 053611 (2002); B. B. Baizakov, V. V. Konotop and M. Salerno, J. Phys. B 35, 5105 (2002).

[4] E. A. Ostrovskaya and Y. S. Kivshar, Phys. Rev. Lett. 90, 160407 (2003); Opt. Exp. 12, 19 (2004).

[5] B. Eiermann, Th. Anker, M. Albiez, M. Taglieber, P. Treutlein, K.-P. Marzlin, and M. K. Oberthaler, Phys. Rev. Lett. 92, 230401 (2004).

[6] B. A. Malomed, Z. H. Wang, P. L. Chu, and G. D. Peng, J. Opt. Soc. Am. B 16, 1197 (1999).

[7] G. L. Alfimov, V. V. Konotop, and M. Salerno, Europhys. Lett. 58, 7 (2002); V. A. Brazhnyi and V. V. Konotop, Mod. Phys. Lett. B 18, 627 (2004).

[8] F. Dalfovo, S. Giorgini, L. P. Pitaevskii, and S. Stringari, Rev. Mod. Phys. 71, 463 (1999).

[9] A. Trombettoni and A. Smerzi, Phys. Rev. Lett. 86, 2353-2356 (2001); G. L. Alfimov, P. G. Kevrekidis, V. V. Konotop, and M. Salerno, Phys. Rev. E 66, 046608 (2002).

[10] G. J. Milburn, J. Corney, E. M. Wright, and D. F. Walls, Phys. Rev. A 55, 4318-4324 (1997); A. Smerzi, S. Fantoni, S. Giovanazzi, and S. R. Shenoy, Phys. Rev. Lett. 79, 4950-4953 (1997); J. Ruostekoski and D. F. Walls, Phys. Rev. A 58, R50-R53 (1998); S. Raghavan, A. Smerzi, S. Fantoni, and S. R. Shenoy, ibid. 59, 620-633 (1999); L. Salasnich, A. Parola, and L. Reatto, ibid. 60, 4171-4174 (1999); E. A. Ostrovskaya, Y. S. Kivshar, M. Lisak, B. Hall, F. Cattani, and D. Anderson, ibid. 61, 031601 (2000); J. E. Williams, ibid. 64, 013610 (2001); W. H. Aschbacher, J. Frohlich, G. M. Graf, K. Schnee, and M. Troyer, J. Math. Phys. 43, 3879 (2002); L. Salasnich, A. Parola, and L. Reatto, J. Phys. B - At. Mol. Opt. Phys. 35, 3205 (2002); P. Coullet and N. Vandenberghe, ibid. 35, 1593-1612 (2002); T. G. Tiecke, M. Kemmann, C. Buggle, I. Shvarchuck, W. von Klitzing, and J. T. M. Walraven, J. Opt. B - Quant. Semicl. Opt. 5, S119 (2003); E. Sakellari, N. P. Proukakis, and C. S. Adams, J. Phys. B - At. Mol. Opt. Phys. 37, 3681 (2004); R. K. Jackson and M. I. Weinstein, J. Stat. Phys. 116, 881 (2004); M. Jaaskelainen and P. Meystre, Phys. Rev. A 71, 043603 (2005); E. Infeld, P. Ziń, J. Gocałek, and M. Trippenbach, Phys. Rev. E 74, 026610 (2006); Q.-X. Yuan and G.-H. Ding, Phys. Lett. A 344, 156 (2005); G. Theocharis, P. G. Kevrekidis, D. J. Frantzeskakis, and P. Schmelcher, Phys. Rev. E 74, 056608 (2006); M. Anderlini, J. Sebby-Strabley, J. Kruse, J. V. Porto, and W. D. Phillips, J. Phys. B - At. Mol. Opt. Phys. 39, S199 (2006).

[11] W. Mak, B. A. Malomed, and P. L. Chu, Phys. Rev. E 55, 6134 (1997); ibid. 57, 1092 (1998).

[12] R. Driben, B. A. Malomed, and P. L. Chu, J. Phys. B: At. Mol. Opt. Phys. 39, 2455 (2006); L. Albuch and B. A. Malomed, Mathematics and Computers in Simulation 74, 361 (2007).

[13] P. G. Kevrekidis, Z. G. Chen, B. A. Malomed, D. J. Frantzeskakis, and M. I. Weinstein, Phys. Lett. A 340,275 (2005).

[14] P. L. Chu, B. A. Malomed, and G. D. Peng, J. Opt. Soc. Am. B 10, 1379 (1993); N. Akhmediev and A. Ankiewicz, Phys. Rev. Lett. 70, 2395 (1993); B. A. Malomed, I. Skinner, P. L. Chu, and G. D. Peng, Phys. Rev. E 53, 4084 (1996).

[15] J. Yang, B. A. Malomed, and D. J. Kaup, Phys. Rev. Lett. 83, 1958-1961 (1999); A. R. Champneys, B. A. Malomed, J. Yang, and D. J. Kaup, Physica D 152-153, 340 (2001).

[16] W. C. K. Mak, P. L. Chu and B. A. Malomed, J. Opt. Soc. Am. B 15, 1685 (1998); Phys. Rev. E 69, 066610 (2004).

[17] A. Gubeskys and B. A. Malomed, Eur. Phys. J. D 28, 283 (2004).

[18] A. V. Buryak and A.A. Akhmediev, J. Opt. Soc. Am. B 11, 804 (1994); A.B. Aceves and M. Santagiustina, Phys. Rev. E 56, 1113 (1997).

[19] S. Inouye, M. R. Andrews, J. Stenger, H. J. Miesner, D. M. Stamper-Kurn, and W. Ketterle, Nature 392,51 (1998); E. A. Donley, N. R. Claussen, S. L. Cornish, J. L. Roberts, E. A. Cornell, and C. E. Wieman, ibid. 412, 295 (2001).

[20] A. Locatelli, D. Modotto, D. Paloschi, and C. De Angelis, Opt. Commun. 237, 97 (2004).

[21] V. M. Pérez-García, H. Michinel, and H. Herrero, Phys. Rev. A 57, 3837 (1998); L. Salasnich, Laser Phys. 12, 198 (2002); L. Salasnich, A. Parola, and L. Reatto, Phys. Rev. A 65, 043614 (2002); A. E. Muryshev, G. V. Shlyapnikov, W. Ertmer, K. Sengstock, and M. Lewenstein, Phys. Rev. Lett. 89, 110401 (2002); Y. B. Band, I. Towers, and B. A. Malomed, Phys. Rev. A 67, 023602 (2003); S. De Nicola, B. A. Malomed, and R. Fedele, Phys. Lett. A 360, 164 (2006). 
[22] K. E. Strecker, G. B. Partridge, A. G. Truscott and R. G. Hulet, Nature 417, 150 (2002); L. Khaykovich, F. Schreck, G. Ferrari, T. Bourdel, J. Cubizolles, L. D. Carr, Y. Castin, and C. Salomon, Science 256, 1290 (2002).

[23] C. J. Myatt, E. A. Burt, R. W. Ghrist, E. A. Cornell, and C. E. Wieman, Phys. Rev. Lett. 78, 586 (1997); H. Pu and N. P. Bigelow, ibid. 80, 1130 (1998); D. S. Hall, M. R. Matthews, J. R. Ensher, C. E. Wieman, and E. A. Cornell, ibid. 81, 1539 (1998).

[24] R. J. Ballagh, K. Burnett, and T. F. Scott, Phys. Rev. Lett. 78, 1607 (1997).

[25] J. Williams, R. Walser, J. Cooper, E. Cornell, and M. Holland, Phys. Rev. A 59, R31 (1999); P. Öhberg and S. Stenholm, ibid. 59, 3890 (1999); D. T. Son and M. A. Stephanov, ibid. 65, 063621 (2002); S. D. Jenkins and T. A. B. Kennedy, ibid. 68053607 (2003); A. Kuklov, N. Prokof'ev, and B. Svistunov, ibid. 69, 025601 (2004); Q.-H. Park and J. H. Eberly, ibid. 70, 021602(R) (2004); I. M. Merhasin, B. A. Malomed, and R. Driben, J. Phys. B: At. Mol. Opt. Phys. 38, 877 (2005).

[26] K. M. Hilligsøe, M. K. Oberthaler, and K.-P. Marzlin1, Phys. Rev. A 66, 063605 (2002); D. E. Pelinovsky, A. A. Sukhorukov and Y. S. Kivshar, Phys. Rev. E 70, 036618 (2004).

[27] T. Mayteevarunyoo and B. A. Malomed, Phys. Rev. A 74, 033616 (2006).

[28] M. G. Vakhitov and A. A. Kolokolov, Sov. J. Radiophys. Quantum Electr. 16, 783 (1973).

[29] P. J. Y. Louis, E. A. Ostrovskaya, C. M. Savage, and Y. S. Kivshar, Phys. Rev. A 67, 013602 (2003).

[30] V. S. Shchesnovich, B. A. Malomed, and R. A. Kraenkel, Physica D 188, 213-240 (2004); V. A. Shchesnovich and S. B. Cavalcanti, Phys. Rev. A 71, 023607 (2005); J. Phys. A - Math. Gen. 38, 6917-6938 (2005).

[31] A. A. Sukhorukov and Y. S. Kivshar. Phys. Rev. Lett. 97, 233901 (2006).

[32] Y. Tsofe and B. A. Malomed, In: Proceedings of SPIE, vol. 6604, article 660419 (2007).

[33] S. V. Manakov, Sov. Phys. JETP 38, 248 (1974).

[34] M. V. Tratnik and J. E. Sipe, Phys. Rev. A 38, 2011 (1988).

[35] A. Amoruso, I. Meccoli, A. Minguzzi, and M. P. Tosi, Eur. Phys. J. D 8, 361 (2000); R. Roth and H. Feldmeier, J. Phys. B - At. Mol. Opt. Phys. 34, 4629 (2001); P. Capuzzi, A. Minguzzi, and M. P. Tosi, Phys. Rev. A 67, 053605 (2003); S. K. Adhikari, ibid. 70, 043617 (2004); ibid. A 72, 0536082005 ; J. Phys. B - At. Mol. Opt. Phys. 38, 3607 (2005); Eur. Phys. J. D 40, 157 (2005); S. K. Adhikari and B. A. Malomed, Phys. Rev. A 74, 053620 (2006). 